\title{
Systematic Review \\ Exposure to Metal Mixtures in Association with Cardiovascular Risk Factors and Outcomes: A Scoping Review
}

\author{
Gyeyoon Yim *, Yuting Wang, Caitlin G. Howe ${ }^{+} \mathbb{i}$ and Megan E. Romano ${ }^{+}$ \\ Department of Epidemiology, Geisel School of Medicine at Dartmouth, Lebanon, NH 03756, USA; \\ yuting.wang.gr@dartmouth.edu (Y.W.); caitlin.g.howe@dartmouth.edu (C.G.H.); \\ megan.e.romano@dartmouth.edu (M.E.R.) \\ * Correspondence: gyeyoon.yim@dartmouth.edu \\ + These authors contributed equally to this work.
}

check for

updates

Citation: Yim, G.; Wang, Y.; Howe, C.G.; Romano, M.E. Exposure to Metal Mixtures in Association with Cardiovascular Risk Factors and Outcomes: A Scoping Review. Toxics 2022, 10, 116. https://doi.org/ $10.3390 /$ toxics 10030116

Academic Editors: Christopher Kassotis and Allison Phillips

Received: 17 January 2022

Accepted: 26 February 2022

Published: 1 March 2022

Publisher's Note: MDPI stays neutral with regard to jurisdictional claims in published maps and institutional affiliations.

Copyright: (C) 2022 by the authors. Licensee MDPI, Basel, Switzerland. This article is an open access article distributed under the terms and conditions of the Creative Commons Attribution (CC BY) license (https:// creativecommons.org/licenses/by/ $4.0 /)$.
Abstract: Since the National Institute of Environmental Health Sciences (NIEHS) declared conducting combined exposure research as a priority area, literature on chemical mixtures has grown dramatically. However, a systematic evaluation of the current literature investigating the impacts of metal mixtures on cardiovascular disease (CVD) risk factors and outcomes has thus far not been performed. This scoping review aims to summarize published epidemiology literature on the cardiotoxicity of exposure to multiple metals. We performed systematic searches of MEDLINE (PubMed), Scopus, and Web of Science to identify peer-reviewed studies employing statistical mixture analysis methods to evaluate the impact of metal mixtures on CVD risk factors and outcomes among nonoccupationally exposed populations. The search was limited to papers published on or after 1998, when the first dedicated funding for mixtures research was granted by NIEHS, through 1 October 2021. Twentynine original research studies were identified for review. A notable increase in relevant mixtures publications was observed starting in 2019. The majority of eligible studies were conducted in the United States $(n=10)$ and China $(n=9)$. Sample sizes ranged from 127 to 10,818 . Many of the included studies were cross-sectional in design. Four primary focus areas included: (i) blood pressure and/or diagnosis of hypertension $(n=15)$, (ii) risk of preeclampsia $(n=3)$, (iii) dyslipidemia and/or serum lipid markers $(n=5)$, and (iv) CVD outcomes, including stroke incidence or coronary heart disease $(n=8)$. The most frequently investigated metals included cadmium, lead, arsenic, and cobalt, which were typically measured in blood $(n=15)$. The most commonly utilized multipollutant analysis approaches were Bayesian kernel machine regression (BKMR), weighted quantile sum regression (WQSR), and principal component analysis (PCA). To our knowledge, this is the first scoping review to assess exposure to metal mixtures in relation to CVD risk factors and outcomes. Recommendations for future studies evaluating the associations of exposure to metal mixtures with risk of CVDs and related risk factors include extending environmental mixtures epidemiologic studies to populations with wider metals exposure ranges, including other CVD risk factors or outcomes outside hypertension or dyslipidemia, using repeated measurement of metals to detect windows of susceptibility, and further examining the impacts of potential effect modifiers and confounding factors, such as fish and seafood intake.

Keywords: metal mixtures; cardiovascular diseases; blood pressure; hypertension; preeclampsia; dyslipidemia

\section{Introduction}

Cardiovascular diseases (CVDs), a group of heart and vascular diseases, are the leading causes of morbidity and mortality worldwide, contributing to an estimated 18 million deaths each year [1]. While poor nutrition and physical inactivity have been identified as main contributors to CVDs [2], growing evidence also points to the possible roles of environmental metals and metalloids (collectively referred to as "metals" hereafter) in the 
development of CVDs. Metals persist in the environment [3] and may have detrimental health effects, even at the low concentrations observed in many parts of the world [4]. Sources of exposure for different metals vary, but include drinking water, consuming contaminated food, inhalation of polluted air, and occupational activities (e.g., mining, smelting, and foundries) [3,5]. Co-exposure to more than one metal is common; it is therefore important to understand the potential joint effects of multiple metals on health [5].

Studies using rodent models have demonstrated that exposure to certain metals, such as arsenic (As), cadmium (Cd), chromium ( $\mathrm{Cr})$, and lead $(\mathrm{Pb})$, induces heart dysfunction [6] and hypertension [7]. Oxidative stress [8], altered regulation of endocrine and endothelial vascular functions [9-12], and epigenetic pathways [13-16] have been identified as possible biological mechanisms. A substantial body of epidemiological evidence has also reported associations between metal exposures and CVD or related outcomes, with three recent reviews summarizing these findings $[4,17,18]$. However, prior studies have predominantly investigated metals individually.

Humans are simultaneously exposed to thousands of environmental toxicants every day, and these environmental chemicals may be correlated and interact with each other [19]. Recognizing that most individuals are exposed to complex mixtures of chemicals, the National Institute of Environmental Health Sciences (NIEHS) has encouraged the field to move towards systematically addressing the impacts of combined exposures, since its first dedicated funding mechanism for studying mixtures in 1998 [20]. More recently, the NIEHS has held several mixtures workshops and developed the Powering Research through Innovative Methods for Mixtures in Epidemiology (PRIME) Program to enhance understanding of complex exposures, prioritize mixture research goals, and recommend research collaborations across multidisciplinary settings [21-23]. As a result, several novel statistical methods have been developed for evaluating the effects of environmental chemical mixtures on health outcomes, including Bayesian kernel machine regression (BKMR) [24], weighted quantile sum regression (WQSR) [25], and quantile-based g-computation [26]. However, to our knowledge, no study has yet reviewed epidemiologic studies applying these approaches to investigate how metals impact cardiovascular risk factors and outcomes in the context of multipollutant exposures.

We therefore present a scoping review of the emerging evidence for cardiovascular impacts of metal mixtures. Our objectives were to describe key gaps in our current scientific understanding of metal mixture impacts on cardiovascular risk factors and outcomes and to guide future investigators as they design and conduct studies on this topic or refine topics for future systematic reviews. We describe recent studies in this area, synthesize common themes, and suggest future directions for metal mixtures research.

\section{Methods}

\subsection{Search Strategy}

This review was performed in accordance to the Preferred Reporting Items for Systematic Reviews and Meta-Analyses Extension for Scoping Reviews (PRISMA-ScR) [27]. The protocol was prepared a priori and registered with the Open Science Framework [28].

To identify observational studies that examined the associations of exposure to metal mixtures with cardiovascular risk factors and outcomes applying multipollutant approaches, we developed a comprehensive search strategy with assistance from an experienced librarian. The following databases were searched: MEDLINE (PubMed), Scopus, and Web of Science. The search strategy was developed primarily for MEDLINE (PubMed), with adapted searches for the other databases. A detailed description of the search strategy can be found in Table S1. A literature search was conducted in each database, restricting to papers published between 1998, which is when the NIEHS first funded mixtures grants [20], through 1 October 2021. No language restriction was imposed. Metals were included as specific text-word terms in our search to enhance sensitivity, adding Medical Subject Headings (MeSH) for trace elements. Key search terms for the outcomes were also developed using both MeSH terms to compile synonyms for cardiovascular risk factors 
and outcomes, such as "cardiometabolic risk factors" [Mesh] or "vascular diseases" [Mesh], and more specific text-word terms to increase sensitivity, including stroke, dyslipidemia, and preeclampsia. We further included the commonly used mixture analysis methods that were introduced by the NIEHS, such as BKMR and WQSR [29]. The search strategies were reviewed by other experienced researchers (M.E.R. and C.G.H.) prior to execution. We also manually checked the reference lists of the eligible research to identify additional pertinent studies.

\subsection{Study Selection}

The search records were pooled in Endnote 20 (Clarivate, Philadelphia, PA, USA), and literature screening was carried out using Rayaan (available online: https:/ /www.rayyan. ai/ (accessed on 15 October 2021)). We used the Populations, Exposures, Comparators, and Outcomes (PECO) framework to formulate eligibility criteria (Table 1) [30]. After deduplication performed in Endnote, titles and abstracts were screened for relevance (G.Y.). For the studies deemed potentially eligible, at least two of the four investigators (C.G.H., M.E.R., Y.W., and G.Y.) independently evaluated the full-text papers for studies that had been pre-screened to determine eligibility. Studies were included if they fulfilled the following prespecified eligibility criteria: the study: (1) was conducted in humans; (2) was an original research study published in a peer-reviewed journal; (3) used a cohort, crosssectional, case-control, or panel study design; (4) evaluated at least three or more metals, thus meeting the NIEHS definition of a mixture [31]; (5) measured metals at the individual level, quantified in human biological samples; and (6) used a multipollutant approach rather than traditional regression. Papers were excluded if: (1) they did not describe an original research study (e.g., review, editorial, case report, case series, or non-research letter); (2) the study was experimental or used an ecologic study design; (3) the study focused on prediction models, rather than hypothesis-driven approaches; (4) the unit of analysis was not a study participant (e.g., studies based on spatially interpolated data); (5) metal exposures were estimated using surrogate measures (e.g., by diet, job title, or proximity to contamination sources); (6) the study population was occupationally exposed to metals; (7) cardiovascular risk factors or outcomes were not measured; or (8) the primary outcomes were cardiovascular specific mortality or hospital admission outcomes (due to the possibility of including competing risks [32]). In a few cases, more than one publication from the same cohort/study population was identified; these studies were considered eligible for inclusion if different metal combinations, cardiovascular risk factors/outcomes, or mixtures analysis methods were used. Any disagreements among the four screeners about study eligibility were resolved by consensus discussion.

Table 1. Populations, Exposures, Comparators, and Outcomes (PECO) statement.

\begin{tabular}{|c|c|c|c|}
\hline Populations & Exposures & Comparators & Outcomes \\
\hline $\begin{array}{l}\text { Healthy humans without } \\
\text { restrictions based on age, sex, } \\
\text { race, or country. Studies } \\
\text { which focused on ill patients } \\
\text { and occupational studies } \\
\text { were excluded }\end{array}$ & $\begin{array}{l}\text { Exposure to metals, including } \\
\text { both toxic and essential } \\
\text { elements. To be included, a } \\
\text { minimum of three metals } \\
\text { needed to have been } \\
\text { considered and exposures } \\
\text { must have been measured at } \\
\text { the individual level in human } \\
\text { biological samples (e.g., } \\
\text { serum, urine, or toenails) }\end{array}$ & $\begin{array}{l}\text { The comparators differed } \\
\text { across studies depending on } \\
\text { the mixture analysis } \\
\text { methods used }\end{array}$ & $\begin{array}{l}\text { Cardiovascular risk factors or } \\
\text { outcomes, including stroke, } \\
\text { myocardial infarction, } \\
\text { coronary heart disease, } \\
\text { hypertension, dyslipidemia, } \\
\text { or pregnancy hypertension. } \\
\text { Outcomes could be either } \\
\text { self-reported, extracted from } \\
\text { medical records, or based on } \\
\text { investigator } \\
\text { collected measurements }\end{array}$ \\
\hline
\end{tabular}

\subsection{Data Extraction}

Data on the following characteristics were extracted from the included studies into a premade Google Sheets form: first author, year of publication, study location, study design, 
sample size, metals evaluated, exposure biomarkers, concentration of metals measured in biospecimens (i.e., range, median, or mean), outcomes evaluated, mixture analysis method(s) applied, covariates, and a summary of the study's main findings (Table 2). Some mixture analysis methods provide quantitative risk estimates (e.g., WQSR), but not all do (e.g., regression trees). Where a quantitative effects estimate was available, we report it in our summary table, but qualitative descriptions of associations were necessary for some methods. Four investigators (C.G.H., M.E.R., Y.W., and G.Y.) independently performed data extraction. Two investigators were assigned to each paper, and discrepancies in extracted information were resolved in discussion with a third reviewer. As a scoping review, we did not include a quality evaluation of the included studies [33,34].

\subsection{Data Synthesis and Analysis}

We reviewed and synthesized the evidence separately for each cardiovascular risk factor or outcome. We were not able to perform a meta-analysis, because no method currently exists that can quantitatively evaluate results from studies using different multipollutant approaches. Instead, all eligible studies were summarized narratively. 
Table 2. Studies included in the review.

\begin{tabular}{|c|c|c|c|c|c|c|c|c|c|}
\hline Source & $\begin{array}{c}\text { Study } \\
\text { Location a }\end{array}$ & $\begin{array}{l}\text { Study } \\
\text { Design }\end{array}$ & $\begin{array}{c}\text { Study } \\
\text { Population }{ }^{b}\end{array}$ & $\begin{array}{l}\text { Metals } \\
\text { Included }\end{array}$ & $\begin{array}{c}\text { Exposure } \\
\text { Matrix }\end{array}$ & $\begin{array}{l}\text { Outcome(s) } \\
\text { Studied }\end{array}$ & $\begin{array}{l}\text { Mixture Analysis } \\
\text { Method(s) }\end{array}$ & Covariates & Summary of Main Findings \\
\hline \multicolumn{10}{|c|}{ BP and hypertension $(n=15)$} \\
\hline Park et al. 2017 & USA & CS & $\begin{array}{l}9664 \text { adults } \\
\text { (4911 females/ } \\
4753 \text { males) }\end{array}$ & $\begin{array}{l}\mathrm{Pb}, \mathrm{Cd}, \mathrm{Hg}, \mathrm{Sb}, \\
\mathrm{As}, \mathrm{Ba}, \mathrm{Co}, \mathrm{Cs}, \\
\text { Mo, Tl, W, } \\
\text { and U }\end{array}$ & $\begin{array}{l}\text { Blood and } \\
\text { urine }\end{array}$ & $\begin{array}{l}\text { SBP, DBP, and } \\
\text { hypertension }\end{array}$ & ERS (AENET-I) & $\begin{array}{l}\text { Age, sex, race/ethnicity, education, } \\
\text { smoking status, and BMI }\end{array}$ & $\begin{array}{l}\text { - } \quad \text { ERS based on AENET-I performed } \\
\text { better than other approaches and } \\
\text { included Cd, Co, and Ba. } \\
\text { ERS showed significant } \\
\text { associations with SBP, DBP, and } \\
\text { hypertension }\end{array}$ \\
\hline Wang et al. 2018 & USA & CS & $\begin{array}{l}9537 \text { adults } \\
\text { (4841 females/ } \\
4696 \text { males) }\end{array}$ & $\begin{array}{l}\mathrm{Cd}, \mathrm{Pb}, \mathrm{Hg}, \mathrm{Sb}, \\
\mathrm{As}, \mathrm{Ba}, \mathrm{Co}, \mathrm{Cs}, \\
\text { Mo, Tl, W, } \\
\text { and U }\end{array}$ & $\begin{array}{l}\text { Blood and } \\
\text { urine }\end{array}$ & Hypertension & $\begin{array}{l}\text { ERS (Adaptive } \\
\text { Elastic Net) }\end{array}$ & $\begin{array}{l}\text { Age, sex, race/ethnicity, education, } \\
\text { smoking status, physical activity, } \\
\text { and NHANES cycles }\end{array}$ & $\begin{array}{l}\text { - ERS included seven main effects } \\
\text { (blood } \mathrm{Pb}, \mathrm{Cd} \text {, and } \mathrm{Hg} \text {, and urinary } \\
\text { monomethylarsonic acid, Ba, Hg, } \\
\text { and } \mathrm{Tl} \text { ), four squared terms, and } \\
\text { seven pairwise interactions } \\
\text { - The association between ERS and } \\
\text { hypertension was significant ( } \mathrm{p}< \\
\text { 0.05) }\end{array}$ \\
\hline Kupsco et al. 2019 & $\begin{array}{l}\text { Mexico City, } \\
\text { Mexico }\end{array}$ & $\mathrm{CO}$ & $\begin{array}{l}548 \text { mother- } \\
\text { child pairs } \\
\text { (272 females / } \\
276 \text { males) }\end{array}$ & $\begin{array}{l}\mathrm{As}, \mathrm{Cd}, \mathrm{Co}, \mathrm{Cr}, \\
\mathrm{Cs}, \mathrm{Cu}, \mathrm{Mn}, \mathrm{Pb}, \\
\mathrm{Sb}, \mathrm{Se} \text {, and } \mathrm{Zn}\end{array}$ & Blood & SBP and DBP & BKMR & $\begin{array}{l}\text { Maternal age, education, } \\
\text { socioeconomic status, parity, } \\
\text { environmental tobacco smoke, and } \\
\text { date of follow-up visit (for HbA1c, } \\
\text { global risk score, non-HDL } \\
\text { cholesterol, SBP and DBP outcomes } \\
\text { only). Birth weight, gestational age, } \\
\text { sex, and pre-pregnancy BMI } \\
\text { included as covariates in } \\
\text { sensitivity analyses }\end{array}$ & $\begin{array}{l}\text { - No effect of the metal mixture on } \\
\text { BP was observed }\end{array}$ \\
\hline $\begin{array}{l}\text { Warembourg et al. } \\
2019\end{array}$ & Europe & $\mathrm{CO}$ & $\begin{array}{l}1277 \text { mother- } \\
\text { child pairs } \\
\text { ( } 580 \text { females/ } \\
697 \text { males) }\end{array}$ & $\begin{array}{l}\text { As, } \mathrm{Cd}, \mathrm{Co}, \mathrm{Cs}, \\
\mathrm{Cu}, \mathrm{Hg}, \mathrm{Mn}, \\
\mathrm{Mo}, \mathrm{Pb}, \text { and } \mathrm{Tl} \\
\text { (out of } \\
89 \text { prenatal } \\
\text { exposures) }\end{array}$ & Blood & SBP and DBP & DSA & $\begin{array}{l}\text { Cohort, maternal age, maternal } \\
\text { education level, maternal } \\
\text { pre-pregnancy BMI, parity, parental } \\
\text { country of birth, child age, child sex, } \\
\text { and child height }\end{array}$ & $\begin{array}{l}\text { The DSA method selected } 5 \text { and } 2 \\
\text { prenatal exposures for SBP and } \\
\text { DBP in childhood, respectively. } \\
\text { - None of these exposures included } \\
\text { metals }\end{array}$ \\
\hline Castiello et al. 2020 & $\begin{array}{l}\text { Granada, } \\
\text { Spain }\end{array}$ & CS & $\begin{array}{l}133 \text { male } \\
\text { adolescents }\end{array}$ & $\begin{array}{l}\mathrm{As}, \mathrm{Cd}, \mathrm{Hg}, \mathrm{Ni}, \\
\mathrm{Pb}, \mathrm{Mn} \text {, and } \mathrm{Cr}\end{array}$ & Urine & $\begin{array}{l}\text { SBP, DBP, } \\
\text { elevated BP, } \\
\text { and PP }\end{array}$ & PCA & Age, serum TG, HDL, LDL, and BMI & $\begin{array}{l}\text { - PC-1 included } \mathrm{Ni}, \mathrm{Cr} \text {, and } \mathrm{Mn} \text {; } \\
\text { PC- } 2 \text { included } \mathrm{As} \text { and } \mathrm{Hg} \text {; and } \\
\text { PC-3 included } \mathrm{Cd} \text { and } \mathrm{Pb} \\
\text { A suggestive association found } \\
\text { between PC-2 and increased SBP }\end{array}$ \\
\hline
\end{tabular}


Table 2. Cont.

\begin{tabular}{|c|c|c|c|c|c|c|c|c|c|}
\hline Source & $\begin{array}{c}\text { Study } \\
\text { Location a }\end{array}$ & $\begin{array}{c}\text { Study } \\
\text { Design }\end{array}$ & $\begin{array}{c}\text { Study } \\
\text { Population }{ }^{b}\end{array}$ & $\begin{array}{l}\text { Metals } \\
\text { Included }\end{array}$ & $\begin{array}{l}\text { Exposure } \\
\text { Matrix }\end{array}$ & $\begin{array}{l}\text { Outcome(s) } \\
\text { Studied }\end{array}$ & $\begin{array}{l}\text { Mixture Analysis } \\
\text { Method(s) }\end{array}$ & Covariates & Summary of Main Findings \\
\hline Desai et al. 2021 & USA & CS & $\begin{array}{l}1642 \text { child or } \\
\text { adolescents } \\
\text { ( } 824 \text { females/ } \\
818 \text { males) }\end{array}$ & $\begin{array}{l}\mathrm{Pb}, \mathrm{Hg}, \mathrm{As} \text {, } \\
\text { and } \mathrm{Cd}\end{array}$ & $\begin{array}{l}\text { Blood and } \\
\text { urine }\end{array}$ & $\begin{array}{l}\text { SBP, DBP } \\
\text { and PP }\end{array}$ & BKMR & $\begin{array}{l}\text { Age, sex, race, BMI, total energy } \\
\text { intake, NHANES cycle, education of } \\
\text { household head, and income to } \\
\text { poverty ratio }\end{array}$ & $\begin{array}{l}\text { - A suggestive inverse association of } \\
\text { the mixture of low-level Pb, Hg, As, } \\
\text { and Cd with DBP was found, but } \\
\text { not with the other outcomes } \\
\text { - No individual association detected } \\
\text { No indication of effect modification } \\
\text { observed }\end{array}$ \\
\hline Everson et al. $2021^{*}$ & USA & CS & $\begin{array}{l}2413 \text { adults } \\
\text { (female to } \\
\text { male ratio } \\
\text { not provided) }\end{array}$ & $\begin{array}{l}\mathrm{Ba}, \mathrm{Cd}, \mathrm{Co}, \mathrm{Cs}, \\
\mathrm{Mo}, \mathrm{Sb}, \mathrm{Tl}, \mathrm{W}, \\
\text { and } \mathrm{Pb}\end{array}$ & $\begin{array}{l}\text { Blood and } \\
\text { urine }\end{array}$ & SBP and DBP & Regression tree & $\begin{array}{l}\text { Age and its squared term, race, sex, } \\
\text { BMI, and smoking status }\end{array}$ & $\begin{array}{l}\text { - Having the higher concentrations } \\
\text { of } \mathrm{Sb}, \mathrm{Cd}, \mathrm{W} \text {, and Pb was associated } \\
\text { with higher predicted SBP } \\
\text { Having the higher concentrations } \\
\text { of Cs and Mo was associated with } \\
\text { lower SBP and DBP } \\
\text { High Sb was particularly predictive } \\
\text { of BP among non-Hispanic white } \\
\text { adults, whereas Cd was } \\
\text { particularly relevant of BP among } \\
\text { non-Hispanic black adults }\end{array}$ \\
\hline Howe et al. 2021 & $\begin{array}{l}\text { Heraklion, } \\
\text { Greece }\end{array}$ & $\mathrm{CO}$ & $\begin{array}{l}176 \text { mother- } \\
\text { child pairs } \\
\text { ( } 78 \text { females/ } \\
98 \text { males) }\end{array}$ & $\begin{array}{l}\mathrm{Mg}, \mathrm{Co}, \mathrm{Se} \\
\mathrm{Mo}, \mathrm{As}, \mathrm{Cd}, \mathrm{Sb}, \\
\text { and } \mathrm{Pb}\end{array}$ & Urine & $\begin{array}{l}\text { SBP, DBP, BP } \\
\text { change, and } \\
\text { elevated BP }\end{array}$ & BKMR & $\begin{array}{l}\text { Maternal age, maternal education, } \\
\text { maternal pre-pregnancy BMI, } \\
\text { maternal smoking during pregnancy, } \\
\text { child's sex, child's age, and } \\
\text { child's height }\end{array}$ & $\begin{array}{l}\text { - } \quad \text { Mo and Co were associated with } \\
\text { increased SBP and DBP at baseline } \\
\text { (age 4). J-shaped associations were } \\
\text { identified } \\
\text { Cd was inversely associated with } \\
\text { DBP at baseline (age 4) } \\
\text { - Co was associated with lower } \\
\text { per-year increases in both SBP and } \\
\text { DBP from ages } 4 \text { to } 11 \\
\text { Mo was associated with lower } \\
\text { per-year increases in DBP from } \\
\text { ages } 4 \text { to } 11 \\
\text { Mg was associated with higher } \\
\text { per-year increases in both SBP and } \\
\text { DBP from ages } 4 \text { to } 11, \text { but not with } \\
\text { BP at baseline (age 4) } \\
\text { Mo and Pb were associated with } \\
\text { BP at age } 11 \text { (J-shaped) } \\
\text { A possible synergistic interaction } \\
\text { between Mo and Pb was shown for } \\
\text { BP at ages } 4 \text { and } 11\end{array}$ \\
\hline
\end{tabular}


Table 2. Cont.

\begin{tabular}{|c|c|c|c|c|c|c|c|c|c|}
\hline Source & $\begin{array}{c}\text { Study } \\
\text { Location a }\end{array}$ & $\begin{array}{c}\text { Study } \\
\text { Design }\end{array}$ & $\begin{array}{c}\text { Study } \\
\text { Population }\end{array}$ & $\begin{array}{l}\text { Metals } \\
\text { Included }\end{array}$ & $\begin{array}{l}\text { Exposure } \\
\text { Matrix }\end{array}$ & $\begin{array}{c}\text { Outcome(s) } \\
\text { Studied }\end{array}$ & $\begin{array}{l}\text { Mixture Analysis } \\
\text { Method(s) }\end{array}$ & Covariates & Summary of Main Findings \\
\hline Kim and Park. 2021 & South Korea & CS & $\begin{array}{l}\text { 10,566 adults } \\
\text { (5843 females/ } \\
4723 \text { males) }\end{array}$ & $\mathrm{Pb}, \mathrm{Hg}$, and $\mathrm{Cd}$ & Blood & $\begin{array}{l}\text { SBP, DBP, and } \\
\text { hypertension }\end{array}$ & WQSR & Age and sex & $\begin{array}{l}\text { - A quartile increase in the WQSR } \\
\text { index was significantly associated } \\
\text { with hypertension risk [OR }=1.29 \\
\text { (95\% CI: } 1.19-1.40)] \\
\text { Pb contributed most to the WQSR } \\
\text { index (weight: } 0.662 \text { compared } \\
\text { with } 0.232 \text { for Hg and } 0.106 \text { for Cd) }\end{array}$ \\
\hline Shih et al. 2021 & Bangladesh & $\mathrm{CO}$ & $\begin{array}{l}491 \text { mother- } \\
\text { child pairs } \\
\text { (242 females/ } \\
249 \text { males) }\end{array}$ & $\begin{array}{l}\mathrm{Al}, \mathrm{As}, \mathrm{Cd}, \mathrm{Cr}, \\
\mathrm{Co}, \mathrm{Cu}, \mathrm{Fe}, \mathrm{Pb}, \\
\mathrm{Mn}, \mathrm{Hg}, \mathrm{Mo}, \\
\mathrm{Ni}, \mathrm{Se}, \mathrm{Sn}, \mathrm{U}, \mathrm{V}, \\
\text { and } \mathrm{Zn}\end{array}$ & $\begin{array}{l}\text { Toenail } \\
\text { samples }\end{array}$ & $\mathrm{BP}$ & $\begin{array}{l}\text { PCA, WQSR, and } \\
\text { BKMR }\end{array}$ & $\begin{array}{l}\text { Maternal age, maternal education, } \\
\text { passive tobacco smoke exposure } \\
\text { during pregnancy, child age, child } \\
\text { sex, and height }\end{array}$ & 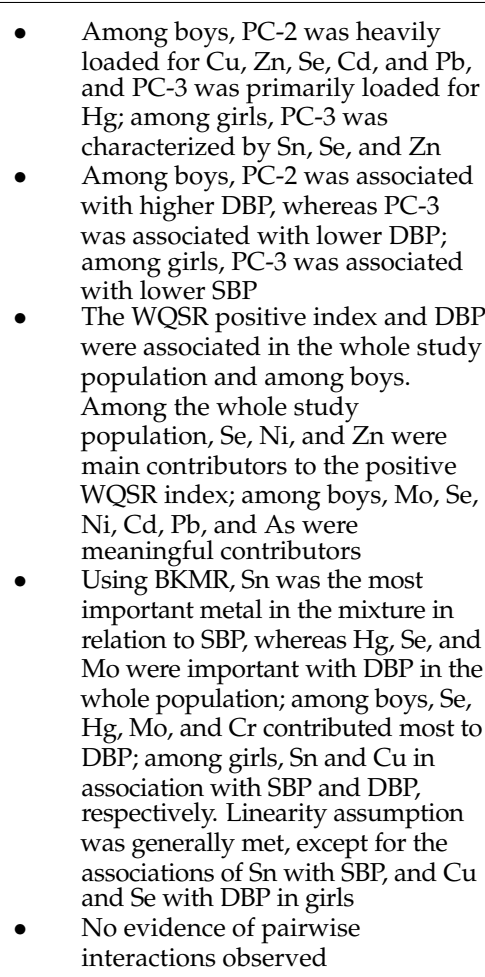 \\
\hline
\end{tabular}


Table 2. Cont.

\begin{tabular}{|c|c|c|c|c|c|c|c|c|c|}
\hline Source & $\begin{array}{c}\text { Study } \\
\text { Location a }\end{array}$ & $\begin{array}{c}\text { Study } \\
\text { Design }\end{array}$ & $\begin{array}{c}\text { Study } \\
\text { Population } b\end{array}$ & $\begin{array}{l}\text { Metals } \\
\text { Included }\end{array}$ & $\begin{array}{l}\text { Exposure } \\
\text { Matrix }\end{array}$ & $\begin{array}{l}\text { Outcome(s) } \\
\text { Studied }\end{array}$ & $\begin{array}{l}\text { Mixture Analysis } \\
\text { Method(s) }\end{array}$ & Covariates & Summary of Main Findings \\
\hline Xu et al. 2021 & USA & CS & $\begin{array}{l}957 \text { adults } \\
\text { (246 females/ } \\
711 \text { males) }\end{array}$ & $\begin{array}{l}\mathrm{Cd}, \mathrm{Pb}, \mathrm{Hg} \text {, } \\
\mathrm{Mn} \text {, and Se }\end{array}$ & Blood & $\begin{array}{l}\text { BP and } \\
\text { hypertension }\end{array}$ & $\begin{array}{l}\text { Quantile } \\
\text { g-computation }\end{array}$ & $\begin{array}{l}\text { Age, sex, race, educational } \\
\text { attainment, and household } \\
\text { income level }\end{array}$ & $\begin{array}{l}\text { A quartile increase in the } \\
\text { mixture was not significantly } \\
\text { associated with the prevalence } \\
\text { of hypertension (OR: } 0.96 ; 95 \% \\
\text { CI: } 0.73-1.27) \\
\text { Mn and Se were positively } \\
\text { weighted (summary OR:1.15), } \\
\text { whereas Pb, } \mathrm{Hg} \text {, and Cd were } \\
\text { negatively weighted (summary } \\
\text { OR: } 0.84 \text { ) within the mixture in } \\
\text { relation to risk of hypertension } \\
\text { Mn }(0.62) \text { and Pb (0.45) had the } \\
\text { greatest proportional positive } \\
\text { or negative contribution to the } \\
\text { joint effect, respectively }\end{array}$ \\
\hline Yao et al. 2021 & USA & CS & $\begin{array}{l}9662 \text { adults } \\
\text { (4910 females/ } \\
4752 \text { males) }\end{array}$ & $\begin{array}{l}\text { As, } \mathrm{Pb}, \mathrm{Cd}, \\
\text { and } \mathrm{Hg}\end{array}$ & $\begin{array}{l}\text { Blood and } \\
\text { urine }\end{array}$ & $\begin{array}{l}\text { SBP, DBP, and } \\
\text { hypertension }\end{array}$ & K-medoids & $\begin{array}{l}\text { Age, gender, ethnicity, education, } \\
\text { smoking status, and BMI }\end{array}$ & $\begin{array}{l}\text { - The k-medoids algorithm } \\
\text { categorized the study } \\
\text { population into } 2 \text { groups } \\
\text { according to either blood or } \\
\text { urinary levels of heavy metals } \\
\text { The "high-exposure" group } \\
\text { based on blood levels was } \\
\text { significantly associated with SBP }\end{array}$ \\
\hline Zhang et al. 2021 & Boston, USA & $\mathrm{CO}$ & $\begin{array}{l}1194 \text { mother- } \\
\text { child pairs } \\
\text { (603 females/ } \\
591 \text { males) }\end{array}$ & $\begin{array}{l}\mathrm{Pb}, \mathrm{Hg}, \mathrm{Cd}, \mathrm{Se} \text {, } \\
\text { and } \mathrm{Mn}\end{array}$ & Blood & SBP and DBP & BKMR & $\begin{array}{l}\text { Maternal age, at delivery, } \\
\text { race/ethnicity, educational level, } \\
\text { pre-pregnancy body mass index, and } \\
\text { cigarette smoking history }\end{array}$ & $\begin{array}{l}\text { - Joint association not found } \\
\text { The hierarchical variable } \\
\text { selection indicated that trace } \\
\text { elements were more strongly } \\
\text { associated with SBP than } \\
\text { heavy metals. Among the } \\
\text { heavy metals, Pb had the } \\
\text { largest conditional PIP; among } \\
\text { the trace elements, Se had the } \\
\text { larger conditional PIP than Mn } \\
\text { In the BKMR individual } \\
\text { analysis, Se and Mn were } \\
\text { inversely associated with child } \\
\text { SBP, but no association } \\
\text { detected for DBP }\end{array}$ \\
\hline
\end{tabular}


Table 2. Cont.

\begin{tabular}{|c|c|c|c|c|c|c|c|c|c|}
\hline Source & $\begin{array}{c}\text { Study } \\
\text { Location a }^{2}\end{array}$ & $\begin{array}{l}\text { Study } \\
\text { Design }\end{array}$ & $\begin{array}{c}\text { Study } \\
\text { Population }{ }^{b}\end{array}$ & $\begin{array}{l}\text { Metals } \\
\text { Included }\end{array}$ & $\begin{array}{c}\text { Exposure } \\
\text { Matrix }\end{array}$ & $\begin{array}{l}\text { Outcome(s) } \\
\text { Studied }\end{array}$ & $\begin{array}{l}\text { Mixture Analysis } \\
\text { Method(s) }\end{array}$ & Covariates & Summary of Main Findings \\
\hline Zhong et al. 2021* & $\begin{array}{l}\text { Tongling, } \\
\text { Maanshan, } \\
\text { and } \\
\text { Chizhou, } \\
\text { China }\end{array}$ & $\mathrm{CO}$ & $\begin{array}{l}1303 \text { adults } \\
\text { (726 females/ } \\
577 \text { males) }\end{array}$ & $\begin{array}{l}\text { As, B, Ba, } \mathrm{Bi} \text {, } \\
\mathrm{Cd}, \mathrm{Co}, \mathrm{Cr}, \mathrm{Cu}, \\
\mathrm{Fe}, \mathrm{Li}, \mathrm{Mg}, \mathrm{Mn} \text {, } \\
\mathrm{Mo}, \mathrm{Rb}, \mathrm{Se}, \mathrm{Sr}, \\
\text { and } \mathrm{Zn}\end{array}$ & Urine & Hypertension & $\begin{array}{l}\mathrm{BKMR}(\mathrm{Cd}, \mathrm{Cu}, \\
\text { Mg, Mo, and } \mathrm{Zn} \\
\text { included) }\end{array}$ & $\begin{array}{l}\text { Age, sex, smoking, drinking, BMI } \\
\text { and BP at baseline }\end{array}$ & $\begin{array}{l}\text { - Significant joint effect of the five } \\
\text { metals on hypertension observed } \\
\text { (at or above their 55th percentile } \\
\text { compared with their median } \\
\text { values) } \\
\text { Three groupPIPs }>0.5 \text {. The } \\
\text { condPIPs of Mo }(0.67) \text { and } \mathrm{Zn}(0.91) \\
\text { were the highest in their groups, } \\
\text { respectively } \\
\text { Cd associated with increased odds } \\
\text { of hypertension, holding other } \\
\text { metals constant at their medians } \\
\text { Potential interaction between Cd } \\
\text { and Zn was observed for elevated } \\
\text { risk of hypertension }\end{array}$ \\
\hline Zuk et al. 2021 & $\begin{array}{l}\text { Quebec, } \\
\text { Canada }\end{array}$ & CS & $\begin{array}{l}759 \text { adults } \\
\text { (447 females/ } \\
312 \text { males) }\end{array}$ & $\begin{array}{l}\mathrm{Cd}, \mathrm{Hg}, \mathrm{Pb} \text {, } \\
\text { and Se (along } \\
\text { with other } \\
\text { POPs) }\end{array}$ & Blood & $\begin{array}{l}\text { BP and } \\
\text { hypertension }\end{array}$ & PCA & $\begin{array}{l}\text { Age, sex, total lipids, smoking status, } \\
\text { and BMI }\end{array}$ & $\begin{array}{l}\text { PC-1 was highly and positively } \\
\text { loaded for all PCBs, OCs, and } \\
\text { moderately loaded on Hg. PC-2 } \\
\text { was moderately loaded on Pb } \\
\text { PC-1 was associated with stage } 2 \\
\text { hypertension }\end{array}$ \\
\hline \multicolumn{10}{|c|}{ Preeclampsia $(n=3)$} \\
\hline $\begin{array}{l}\text { Bommarito et al. } \\
2019^{*}\end{array}$ & Boston, USA & $\mathrm{CO}$ & $\begin{array}{l}28 / 355 \\
\text { pregnant } \\
\text { women }\end{array}$ & $\begin{array}{l}\text { As, Ba, Cd, Cu, } \\
\mathrm{Hg}, \mathrm{Mn}, \mathrm{Mo}, \\
\mathrm{Ni}, \mathrm{Pb}, \mathrm{Se}, \mathrm{Sn}, \\
\text { Tl, Zn, Be, Cr, } \\
\text { U, and W }\end{array}$ & Urine & Preeclampsia & PCA & $\begin{array}{l}\text { Smoking during pregnancy, race, } \\
\text { educational attainment, insurance } \\
\text { status, infant sex, ART, calcium } \\
\text { supplementation, pre-pregnancy } \\
\text { BMI, and gestational age at study } \\
\text { visit }\end{array}$ & $\begin{array}{l}\text { - PC-1 was characterized by } \\
\text { essential metals (Cu, Se, and } \mathrm{Zn}) \text {; } \\
\text { PC-2 characterized by toxic metals } \\
\text { (Cd, Mn, and Pb); and PC-3 } \\
\text { characterized by } \\
\text { seafood-associated metals } \\
\text { No main associations observed } \\
\text { between the three PCs and } \\
\text { preeclampsia } \\
\text { The association between PC-2 and } \\
\text { preeclampsia was significant } \\
\text { among individuals with low levels } \\
\text { of PC- } 1\end{array}$ \\
\hline
\end{tabular}


Table 2. Cont.

\begin{tabular}{|c|c|c|c|c|c|c|c|c|c|}
\hline Source & $\begin{array}{c}\text { Study } \\
\text { Location a }\end{array}$ & $\begin{array}{l}\text { Study } \\
\text { Design }\end{array}$ & $\begin{array}{c}\text { Study } \\
\text { Population }{ }^{b}\end{array}$ & $\begin{array}{c}\text { Metals } \\
\text { Included }\end{array}$ & $\begin{array}{l}\text { Exposure } \\
\text { Matrix }\end{array}$ & $\begin{array}{l}\text { Outcome(s) } \\
\text { Studied }\end{array}$ & $\begin{array}{l}\text { Mixture Analysis } \\
\text { Method(s) }\end{array}$ & Covariates & Summary of Main Findings \\
\hline Wang et al. 2020 & $\begin{array}{l}\text { Taiyuan, } \\
\text { China }\end{array}$ & CC & $\begin{array}{l}427 / 427 \\
\text { pregnant } \\
\text { women }\end{array}$ & $\begin{array}{l}\mathrm{Cr}, \mathrm{Co}, \mathrm{Ni}, \mathrm{As}, \\
\mathrm{Cd}, \mathrm{Sb}, \mathrm{Hg} \text {, } \\
\text { and } \mathrm{Pb}\end{array}$ & Blood & Preeclampsia & WQSR and PCA & $\begin{array}{l}\text { Matched by age, residence area, and } \\
\text { conception time, and adjusted for } \\
\text { education, household monthly } \\
\text { income per capita, gestational age, } \\
\text { and pre-pregnancy BMI }\end{array}$ & $\begin{array}{l}\text { - Individuals with PC-2 scores in the } \\
\text { highest tertile (high loadings for Cr } \\
\text { and As) had increased prevalence } \\
\text { of preeclampsia compared with } \\
\text { those in the lowest tertile (OR: } 1.59 \text {; } \\
95 \% \text { CI: } 1.10-2.31) \\
\text { Individuals with PC-3 scores in the } \\
\text { highest and middle tertiles (high } \\
\text { loadings for Pb and Hg) had } \\
\text { increased prevalence of early onset } \\
\text { preeclampsia compared with those } \\
\text { in the lowest tertile (OR: } 2.19 ; 95 \% \\
\text { CI: } 1.34-3.60 \text { and OR: } 2.48 ; 95 \% \text { CI: } \\
\text { 1.45-4.25 for the middle or highest } \\
\text { tertile vs. lowest tertile, } \\
\text { respectively) } \\
\text { In the WQSR analysis, OR for } \\
\text { tertile sum increase in metals and } \\
\text { preeclampsia: } 1.68 \text { ( } 95 \% \text { CI: } \\
\text { 1.20-2.33); largest weights for Cr } \\
\text { (0.447), Hg (0.216), Pb (0.183), and } \\
\text { As (0.139) }\end{array}$ \\
\hline Liu et al. 2021 & $\begin{array}{l}\text { USA (12 } \\
\text { clinical sites } \\
\text { across the } \\
\text { nation) }\end{array}$ & $\mathrm{CO}$ & $\begin{array}{l}1832 \text { women } \\
\text { in the } \\
\text { longitudinal } \\
\text { analysis, } \\
1688 \text { women } \\
\text { in the cross- } \\
\text { sectional } \\
\text { analysis }\end{array}$ & $\begin{array}{l}\mathrm{Ba}, \mathrm{Cs}, \mathrm{Sb}, \mathrm{Co}, \\
\mathrm{Cu}, \mathrm{Mo}, \mathrm{Se}, \\
\text { and } \mathrm{Zn}\end{array}$ & Blood & $\begin{array}{l}\text { Baseline SBP, } \\
\text { DBP, and rates } \\
\text { of weekly BP } \\
\text { changes over } \\
\text { pregnancy }\end{array}$ & BKMR & $\begin{array}{l}\text { Maternal age at enrollment, maternal } \\
\text { race/ethnicity, maternal educational } \\
\text { achievement, marital status, parity, } \\
\text { self-reported pre-pregnancy BMI, } \\
\text { pre-pregnancy to 1st trimester } \\
\text { moderate to vigorous level of } \\
\text { physical activity, and gestational age } \\
\text { at chemical measurement }\end{array}$ & $\begin{array}{l}\text { - Holding all chemicals at their 90th } \\
\text { percentile was associated with a } \\
1.61 \mathrm{mmHg}(95 \% \text { CI: } 0.41,2.81) \\
\text { higher SBP and a } 1.09 \mathrm{mmHg} \text { ( } 95 \% \\
\text { CI: } 0.10,2.09 \text { ) higher DBP at } \\
\text { baseline compared to holding all } \\
\text { chemicals at their median levels } \\
\text { Accounting for other chemicals } \\
\text { within the mixture, each IQR } \\
\text { increment in Cu was associated } \\
\text { with a } 0.67 \mathrm{mmHg}(95 \% \mathrm{CI}: 0.02, \\
1.32) \text { higher SBP and a } 0.60 \mathrm{mmHg} \\
\text { (95\% CI: } 0.08,1.12) \text { higher DBP at } \\
\text { baseline; each IQR increment in Se } \\
\text { was associated with a 0.67 mmHg } \\
\text { (95\% CI: 0.05, 1.29) higher SBP but } \\
\text { not DBP } \\
\text { No interactions observed }\end{array}$ \\
\hline
\end{tabular}


Table 2. Cont.

\begin{tabular}{|c|c|c|c|c|c|c|c|c|c|}
\hline Source & $\begin{array}{c}\text { Study } \\
\text { Location a }\end{array}$ & $\begin{array}{l}\text { Study } \\
\text { Design }\end{array}$ & $\begin{array}{c}\text { Study } \\
\text { Population }\end{array}$ & $\begin{array}{c}\text { Metals } \\
\text { Included }\end{array}$ & $\begin{array}{c}\text { Exposure } \\
\text { Matrix }\end{array}$ & $\begin{array}{c}\text { Outcome(s) } \\
\text { Studied }\end{array}$ & $\begin{array}{l}\text { Mixture Analysis } \\
\text { Method(s) }\end{array}$ & Covariates & Summary of Main Findings \\
\hline \multicolumn{10}{|c|}{ Dyslipidemia and serum lipid levels $(n=5)$} \\
\hline Park et al. 2014 & USA & CS & $\begin{array}{l}\text { Stage 1: } \\
10,818 \text { adults } \\
\text { (5789 females/ } \\
5029 \text { males) } \\
\text { Stage 2: } \\
4615 \text { adults } \\
\text { (2395 females/ } \\
2220 \text { males) }\end{array}$ & $\begin{array}{l}\mathrm{Pb}, \mathrm{Cd}, \mathrm{Hg}, \\
\text { and As out of } \\
149 \text { pollutants }\end{array}$ & $\begin{array}{l}\text { Blood and } \\
\text { urine }\end{array}$ & $\begin{array}{l}\text { TC, HDL, LDL, } \\
\text { and TG }\end{array}$ & ERS (EWAS) & $\begin{array}{l}\text { Age, gender, race/ethnicity, } \\
\text { education, BMI, and serum } \\
\text { micronutrients }\end{array}$ & $\begin{array}{l}\text { - The EWAS identified } 13 \text { pollutants } \\
\text { associated with total cholesterol, } 9 \\
\text { for HDL, } 5 \text { for LDL and } 27 \text { for } \\
\text { triglycerides } \\
\text { In the multi-pollutant analysis, } \\
\text { blood Pb and Cd were associated } \\
\text { with increased total cholesterol } \\
\text { Blood Pb was also associated with } \\
\text { greater HDL and LDL } \\
\text { Blood Cd was associated with } \\
\text { greater triglycerides. Urinary Cd } \\
\text { was associated with lower HDL } \\
\text { and higher LDL } \\
\text { Hg in blood or urine and urinary } \\
\text { arsenobetaine were associated with } \\
\text { decreased triglycerides } \\
\text { Urinary Sb was associated with } \\
\text { lower HDL }\end{array}$ \\
\hline Kupsco et al. 2019 & $\begin{array}{l}\text { Mexico City, } \\
\text { Mexico }\end{array}$ & $\mathrm{CO}$ & $\begin{array}{l}548 \text { mother- } \\
\text { child pairs } \\
\text { (272 females/ } \\
276 \text { males) }\end{array}$ & $\begin{array}{l}\mathrm{As}, \mathrm{Cd}, \mathrm{Co}, \mathrm{Cr}, \\
\mathrm{Cs}, \mathrm{Cu}, \mathrm{Mn}, \mathrm{Pb}, \\
\mathrm{Sb}, \mathrm{Se} \text {, and } \mathrm{Zn}\end{array}$ & Blood & $\begin{array}{l}\text { Non-HDL } \\
\text { cholesterol, } \\
\text { TG, leptin, } \\
\text { adiponectin }\end{array}$ & BKMR & $\begin{array}{l}\text { Maternal age, education, } \\
\text { socioeconomic status, parity, } \\
\text { environmental tobacco smoke, and } \\
\text { date of follow-up visit (for HbA1c, } \\
\text { global risk score, non-HDL } \\
\text { cholesterol, SBP and DBP outcomes } \\
\text { only). Birth weight, gestational age, } \\
\text { sex, and pre-pregnancy BMI } \\
\text { included as covariates in } \\
\text { sensitivity analyses }\end{array}$ & $\begin{array}{ll} & \text { Higher Se was associated with } \\
\text { lower TG } & \text { Sb and As were associated with } \\
\text { lower leptin } \\
\text { - No interaction among the metals or } \\
\text { non-linear responses detected }\end{array}$ \\
\hline Zhu et al. 2021 & $\begin{array}{l}\text { West Anhui, } \\
\text { China }\end{array}$ & CS & $\begin{array}{l}1013 \text { adults } \\
\text { (552 females/ } \\
461 \text { males) }\end{array}$ & $\begin{array}{l}\mathrm{Sr}, \mathrm{Cd}, \mathrm{Pb}, \mathrm{V}, \\
\mathrm{Al}, \mathrm{Co} \text {, and } \mathrm{Mn}\end{array}$ & Blood & Dyslipidemia & PCA & $\begin{array}{l}\text { Gender, age, education level, per } \\
\text { capita income, BMI, occupation, } \\
\text { smoking, drinking, exercise, and } \\
\text { disease history of hypertension, } \\
\text { diabetes, stroke, and coronary } \\
\text { heart disease }\end{array}$ & $\begin{array}{l}\text { - } \quad \text { PC-1 was represented by V, Al, and } \\
\text { Co; PC-2 (Sr, Cd, and Pb); and PC-3 } \\
\text { (Mn) } \\
\text { PC-2 was positively associated } \\
\text { with the prevalence of } \\
\text { dyslipidemia, whereas PC-1 was } \\
\text { inversely associated } \\
\text { - No association found between Mn } \\
\text { (PC-3) and dyslipidemia }\end{array}$ \\
\hline
\end{tabular}


Table 2. Cont.

\begin{tabular}{|c|c|c|c|c|c|c|c|c|c|}
\hline Source & $\begin{array}{c}\text { Study } \\
\text { Location a }\end{array}$ & $\begin{array}{c}\text { Study } \\
\text { Design }\end{array}$ & $\begin{array}{c}\text { Study } \\
\text { Population }\end{array}$ & $\begin{array}{c}\text { Metals } \\
\text { Included }\end{array}$ & $\begin{array}{c}\text { Exposure } \\
\text { Matrix }\end{array}$ & $\begin{array}{c}\text { Outcome(s) } \\
\text { Studied }\end{array}$ & $\begin{array}{l}\text { Mixture Analysis } \\
\text { Method(s) }\end{array}$ & Covariates & Summary of Main Findings \\
\hline Jiang et al. 2021 & $\begin{array}{l}\text { Hubei, } \\
\text { China }\end{array}$ & $\mathrm{CO}$ & $\begin{array}{l}2947 \text { adults } \\
\text { (1473 females/ } \\
1474 \text { males) }\end{array}$ & $\begin{array}{l}\mathrm{Al}, \mathrm{Sb}, \mathrm{As}, \mathrm{Ba}, \\
\mathrm{Co}, \mathrm{Cu}, \mathrm{Pb} \\
\mathrm{Mn}, \mathrm{Mo}, \mathrm{Ni} \\
\mathrm{Rb}, \mathrm{Se}, \mathrm{Sr}, \mathrm{Tl}, \\
\mathrm{Ti}, \mathrm{V} \text {, and } \mathrm{Zn}\end{array}$ & Blood & $\begin{array}{l}\text { Incident } \\
\text { dyslipidemia }\end{array}$ & PCA & $\begin{array}{l}\text { Age, gender, BMI, education level, } \\
\text { smoking status, drinking status, } \\
\text { physical activity, fasting blood } \\
\text { glucose, eGFR, hypertension, family } \\
\text { history of dyslipidemia, and } \\
\text { measurement batch }\end{array}$ & $\begin{array}{l}\text { PC-1 characterized by combined } \\
\text { exposure to Al, As, Ba, Pb, V, and } \\
\mathrm{Zn} ; \mathrm{PC}-2 \text { ( } \mathrm{Sb}, \mathrm{CO} \text {, and Tl); PC-3 (Cu, } \\
\mathrm{Rb} \text {, and Se); } \mathrm{PC}-4 \text { (Ti and V); and } \\
\text { PC-5 (Ni) } \\
\text { Compared with the first quartile of } \\
\text { the scores of PC-1, the fourth } \\
\text { quartile of PC-1 was associated } \\
\text { with elevated risk of dyslipidemia } \\
\text { (aOR: } 1.40 ; 95 \% \text { CI: } 1.07,1.84 \text { ). PC-1 } \\
\text { also associated with higher risks of } \\
\text { low HDL-C and high LDL-C } \\
\text { PC-3 was associated with the } \\
\text { higher TC risk and lower HDL-C } \\
\text { risk. }\end{array}$ \\
\hline Li et al. $2021^{*}$ & $\begin{array}{l}\text { Hunan, } \\
\text { China }\end{array}$ & CS & $\begin{array}{l}564 \\
\text { (293 females/ } \\
271 \text { males) } \\
\text { and } \\
637 \text { adults } \\
\text { (449 females/ } \\
188 \text { males) } \\
\text { from Shimen } \\
\text { and Huayuan, } \\
\text { respectively }\end{array}$ & $\begin{array}{l}\mathrm{Al}, \mathrm{As}, \mathrm{Ba}, \mathrm{Cd}, \\
\mathrm{Co}, \mathrm{Cr}, \mathrm{Cu}, \mathrm{Fe}, \\
\mathrm{Mn}, \mathrm{Mo}, \mathrm{Ni} \text {, } \\
\mathrm{Rb}, \mathrm{Sb}, \mathrm{Se}, \mathrm{Sn}, \\
\mathrm{Sr} \text {, Ti, Tl, U, W, } \\
\text { V, and Zn }\end{array}$ & $\begin{array}{l}\text { Blood and } \\
\text { urine }\end{array}$ & $\begin{array}{l}\text { The } \\
\text { concentrations } \\
\text { of TG, TC, } \\
\text { HDL-C, and } \\
\text { LDL-C }\end{array}$ & WQSR and BKMR & $\begin{array}{l}\text { Age, gender, BMI, smoke, drink, } \\
\text { physical activity, education, ethnicity, } \\
\text { income level, hypertension, family } \\
\text { history of hyperlipidemia, and eGFR }\end{array}$ & $\begin{array}{l}\text { - WQSR index associated with lipids, } \\
\text { except for HDL-C, for both } \\
\text { positively and negatively } \\
\text { constrained models. } \\
\text { In the grouped WQSR analysis, } \\
\text { essential metals were positively } \\
\text { associated with all lipid markers, } \\
\text { except for HDL-C in Huayuan area. } \\
\text { Toxic metals were negatively } \\
\text { associated with lipids, except for } \\
\text { HDL-C and LDL-C in Huayuan } \\
\text { area. } \\
\text { BKMR suggested no joint effects } \\
\text { - Potential non-linear relationship } \\
\text { was observed between the metal } \\
\text { mixture and TC and LDL-C levels } \\
\text { The associations of exposure to Zn } \\
\text { or Ti with TG levels were } \\
\text { consistently found in both areas, } \\
\text { but the other associations varied by } \\
\text { sites } \\
\text { An inverse U-shaped association of } \\
\text { Fe with LDL-C levels were } \\
\text { detected in Huayuan area } \\
\text { Possible interaction between Zn } \\
\text { and Cd was detected in association } \\
\text { with LDL-C in Huayuan area }\end{array}$ \\
\hline
\end{tabular}


Table 2. Cont.

\begin{tabular}{|c|c|c|c|c|c|c|c|c|c|}
\hline Source & $\begin{array}{c}\text { Study } \\
\text { Location a }\end{array}$ & $\begin{array}{c}\text { Study } \\
\text { Design }\end{array}$ & $\begin{array}{c}\text { Study } \\
\text { Population }{ }^{\mathrm{b}}\end{array}$ & $\begin{array}{l}\text { Metals } \\
\text { Included }\end{array}$ & $\begin{array}{l}\text { Exposure } \\
\text { Matrix }\end{array}$ & $\begin{array}{l}\text { Outcome(s) } \\
\text { Studied }\end{array}$ & $\begin{array}{l}\text { Mixture Analysis } \\
\text { Method(s) }\end{array}$ & Covariates & Summary of Main Findings \\
\hline \multicolumn{10}{|c|}{ CVD outcomes $(n=8)$} \\
\hline $\begin{array}{l}\text { Domingo-Relloso } \\
\text { et al. } 2019\end{array}$ & $\begin{array}{l}\text { Valladolid, } \\
\text { Spain }\end{array}$ & $\mathrm{CO}$ & $\begin{array}{l}1171 \text { adults } \\
\text { (566 females/ } \\
605 \text { males }\end{array}$ & $\begin{array}{l}\mathrm{Sb}, \mathrm{Ba}, \mathrm{Cd}, \mathrm{Cr}, \\
\mathrm{Co}, \mathrm{Cu}, \mathrm{Mo}, \mathrm{V}, \\
\text { and } \mathrm{Zn}\end{array}$ & Urine & $\begin{array}{l}\text { Combined } \\
\text { endpoint for } \\
\text { incident } \\
\text { coronary heart } \\
\text { disease and } \\
\text { stroke }\end{array}$ & BKMR-P & $\begin{array}{l}\text { Sex, education, smoking status, } \\
\text { cumulative smoking dose, urine } \\
\text { cotinine, age, estimated GFR, } \\
\text { residence, HDL cholesterol level, } \\
\text { total cholesterol level, dyslipidemia } \\
\text { treatment, hypertension treatment, } \\
\text { diabetes mellitus of type } 2 \text { and } \\
\text { systolic pressure }\end{array}$ & $\begin{array}{l}\text { - } \quad \mathrm{Cu}, \mathrm{Zn}, \mathrm{Sb}, \mathrm{Cd}, \mathrm{Sr} \text {, and } \mathrm{V} \text { were } \\
\text { associated with cardiovascular } \\
\text { incidence, with Cd and Sb showing } \\
\text { the highest PIPs }\end{array}$ \\
\hline Liberda et al. 2019 & $\begin{array}{l}\text { Quebec, } \\
\text { Canada }\end{array}$ & CS & $\begin{array}{l}535 \text { adults } \\
\text { (299 females/ } \\
236 \text { males) }\end{array}$ & $\begin{array}{l}\text { As, } \mathrm{Pb}, \mathrm{Cd}, \mathrm{Hg} \text {, } \\
\mathrm{Se}, \mathrm{Co}, \mathrm{Cu}, \mathrm{Mo} \text {, } \\
\mathrm{Ni} \text { and } \mathrm{Zn} \text { out } \\
\text { of } 43 \\
\text { contaminants }\end{array}$ & Blood & $\begin{array}{l}\text { Carotid } \\
\text { intima-media } \\
\text { thickness }\end{array}$ & PCA & $\begin{array}{l}\text { Age, sex, smoking status, BMI, SBP, } \\
\text { LDL, Apo-B, triglycerides, TNF- } \alpha \text {, } \\
\text { hs-CRP, and ox-LDL }\end{array}$ & $\begin{array}{l}\text { - Metals were mainly loaded on PC-4 } \\
\text { and PC-5, including Ni, Se, and } \mathrm{Cd} \\
\text { Carotid intima-media thickness } \\
\text { was significantly associated with } \\
\text { PC-5, which was mostly } \\
\text { represented by Ni }\end{array}$ \\
\hline Wen et al. 2019 & $\begin{array}{l}\text { Shenzhen, } \\
\text { China }\end{array}$ & $\mathrm{CC}$ & $\begin{array}{l}1277 / 1277 \\
\text { adults (548 } \\
\text { females/ } \\
729 \text { males for } \\
\text { both controls } \\
\text { and cases) }\end{array}$ & $\begin{array}{l}\mathrm{Al}, \mathrm{As}, \mathrm{Cd}, \mathrm{Co} \\
\mathrm{Cu}, \mathrm{Fe}, \mathrm{Mn} \text {, } \\
\mathrm{Mo}, \mathrm{Se}, \mathrm{Tl} \text {, and } \\
\mathrm{Zn}\end{array}$ & Blood & $\begin{array}{l}\text { First ischemic } \\
\text { stroke }\end{array}$ & PCA & $\begin{array}{l}\text { Matched by age and sex, with } \\
\text { adjustment for BMI, smoking, } \\
\text { alcohol drinking, hypertension, } \\
\text { diabetes, and hyperlipidemia }\end{array}$ & $\begin{array}{l}\text { - The first } \mathrm{PC} \text {, represented by } \mathrm{Al}, \mathrm{Cd} \text {, } \\
\text { and Mn, was positively associated } \\
\text { with risk of ischemic stroke } \\
\text { The second PC, represented by Fe } \\
\text { and Se, was inversely associated } \\
\text { with risk of ischemic stroke }\end{array}$ \\
\hline Xiao et al. 2019 & $\begin{array}{l}\text { Dongfeng, } \\
\text { China }\end{array}$ & $\mathrm{CC}$ & $\begin{array}{l}1035 / 1035 \\
\text { adults ( } 382 \\
\text { females/ } \\
653 \text { males for } \\
\text { both controls } \\
\text { and cases) } \\
\text { for ischemic } \\
\text { stroke; } \\
269 / 269 \\
\text { adults } \\
\text { (112 females/ } \\
157 \text { males) for } \\
\text { hemorrhagic } \\
\text { stroke }\end{array}$ & $\begin{array}{l}\mathrm{Al}, \mathrm{As}, \mathrm{Ba}, \mathrm{Co}, \\
\mathrm{Cu}, \mathrm{Pb}, \mathrm{Mn}, \\
\mathrm{Hg}, \mathrm{Mo}, \mathrm{Ni}^{\prime} \\
\mathrm{Rb}, \mathrm{Se}, \mathrm{Sr}, \mathrm{Tl}, \\
\mathrm{Ti}, \mathrm{W}, \mathrm{V} \text {, and } \\
\mathrm{Zn}\end{array}$ & Blood & Incident stroke & $\begin{array}{l}\text { Elastic net } \\
\text { regression }\end{array}$ & $\begin{array}{l}\text { Matched on age, sex, and blood } \\
\text { sampling date, and adjusted for BMI, } \\
\text { smoking, drinking staus, regular } \\
\text { exercise, family history of stroke, } \\
\text { hyperlipidemia, diabetes mellitus, } \\
\text { and hypertension }\end{array}$ & $\begin{array}{l}\text { Elastic net selected } \mathrm{Cu}, \mathrm{Mo} \text {, and Se } \\
\text { for ischemic stroke, and Rb and Se } \\
\text { for hemorrhagic stroke. The } \\
\text { selected metals were included in } \\
\text { predictive plasma metal scores } \\
\text { Per one IQR increase in predictive } \\
\text { plasma metal scores, the adjusted } \\
\text { OR was } 1.37 \text { (95\% CI: } 1.20,1.56) \text { for } \\
\text { ischemic stroke and } 1.53 \text { (95\% CI: } \\
1.16,2.01) \text { for hemorrhagic stroke }\end{array}$ \\
\hline
\end{tabular}


Table 2. Cont.

\begin{tabular}{|c|c|c|c|c|c|c|c|c|c|}
\hline Source & $\begin{array}{c}\text { Study } \\
\text { Location a }\end{array}$ & $\begin{array}{l}\text { Study } \\
\text { Design }\end{array}$ & $\begin{array}{c}\text { Study } \\
\text { Population b }\end{array}$ & $\begin{array}{l}\text { Metals } \\
\text { Included }\end{array}$ & $\begin{array}{l}\text { Exposure } \\
\text { Matrix }\end{array}$ & $\begin{array}{l}\text { Outcome(s) } \\
\text { Studied }\end{array}$ & $\begin{array}{l}\text { Mixture Analysis } \\
\text { Method(s) }\end{array}$ & Covariates & Summary of Main Findings \\
\hline Cabral et al. 2021 & $\begin{array}{l}\text { Potsdam, } \\
\text { Germany }\end{array}$ & $\mathrm{CCO}$ & $\begin{array}{l}2087 \text { adults } \\
\text { (1304 females/ } \\
783 \text { males) }\end{array}$ & $\begin{array}{l}\mathrm{Mn}, \mathrm{Fe}, \mathrm{Cu}, \mathrm{Zn} \text {, } \\
\mathrm{I} \text {, and Se }\end{array}$ & Blood & $\begin{array}{l}\text { CVD outcomes } \\
\text { (incident MI } \\
\text { and stroke) }\end{array}$ & PCA & $\begin{array}{l}\text { Age, sex, education, BMI, waist } \\
\text { circumference, smoking status, } \\
\text { overall leisure-time physical activity, } \\
\text { alcohol consumption, prevalent } \\
\text { hypertension, anti-hypertensive and } \\
\text { lipid-lowering medication, vitamin } \\
\text { and mineral preparations, and } \\
\text { dietary quality }\end{array}$ & $\begin{array}{l}\text { - PC-1 was mainly related to higher } \\
\text { concentrations of } \mathrm{Mn}, \mathrm{Fe} \text {, and } \mathrm{Zn} \text {, } \\
\text { whereas PC-2 to } \mathrm{Cu}, \mathrm{I} \text {, and Se } \\
\text { Only PC-2 was associated with risk } \\
\text { of developing CVD }\end{array}$ \\
\hline Liu et al. 2021 & $\begin{array}{l}\text { Nanjing, } \\
\text { China }\end{array}$ & $\mathrm{CC}$ & $\begin{array}{l}127 / 183 \\
\text { adults } \\
\text { (30 females/ } \\
97 \text { males for } \\
\text { cases; } \\
46 \text { females/ } \\
137 \text { males for } \\
\text { controls) }\end{array}$ & $\begin{array}{l}\mathrm{Mo}, \mathrm{Tl}, \mathrm{Cu}, \mathrm{Cs}, \\
\mathrm{Ba}, \mathrm{Pb}, \mathrm{Cr}, \mathrm{Mn}, \\
\mathrm{Co} \text {, and } \mathrm{Ni}\end{array}$ & Blood & $\mathrm{AD}$ & BKMR and WQSR & $\begin{array}{l}\text { Matched by age and sex, with } \\
\text { adjustments for BMI, education level, } \\
\text { smoking status, drinking status, BP, } \\
\text { history of hypertension, subtype of } \\
\text { AD, and the WBC count }\end{array}$ & $\begin{array}{l}\text { - The WQSR analysis suggested } \\
\text { elevated risk of AD per every unit } \\
\text { increase in the metal mixture index } \\
\text { (coefficient }=3.49,95 \% \text { CI: } 2.25, \\
5.28 \text {, with } \mathrm{Cu}, \mathrm{Ni} \text {, and Mo as the } \\
\text { main contributors } \\
\text { The BKMR analysis indicated a } \\
\text { significantly positive trend for the } \\
\text { association of the metal mixture } \\
\text { with } \mathrm{AD} \text {, with } \mathrm{Cu} \text { and Mo showing } \\
\text { the greatest PIPs }\end{array}$ \\
\hline Yang et al. 2021* & $\begin{array}{l}\text { Wuhan, } \\
\text { China }\end{array}$ & $\begin{array}{l}\text { Panel } \\
\text { study }\end{array}$ & $\begin{array}{l}127 \text { adults } \\
\text { (90 females/ } \\
37 \text { males) }\end{array}$ & $\begin{array}{l}\mathrm{Al}, \mathrm{Sb}, \mathrm{As}, \mathrm{Ba}, \\
\mathrm{Cd}, \mathrm{Cr}, \mathrm{Co}, \mathrm{Cu}, \\
\mathrm{Fe}, \mathrm{Pb}, \mathrm{Mn}, \\
\mathrm{Mo}, \mathrm{Ni}, \mathrm{Rb}, \mathrm{Se}, \\
\mathrm{Sr}, \mathrm{Tl}, \mathrm{Sn}, \mathrm{Ti}, \mathrm{W}, \\
\mathrm{U}, \mathrm{V} \text {, and } \mathrm{Zn}\end{array}$ & Urine & $\begin{array}{l}\text { Arterial } \\
\text { stiffness of } \\
\text { peripheral } \\
\text { arteries }\end{array}$ & LASSO & $\begin{array}{l}\text { Age, sex, BMI, smoking status, } \\
\text { drinking status, education, physical } \\
\text { activity, hypertension, } \\
\text { hyperlipidemia, diabetes, heart rate, } \\
\text { and community }\end{array}$ & $\begin{array}{l}\text { - Ti and Co were identified as the } \\
\text { important predictors of ABI } \\
\text { In a stratified analysis, the inverse } \\
\text { associations between urinary } \\
\text { metals and ABI was more } \\
\text { pronounced among never-smokers }\end{array}$ \\
\hline
\end{tabular}

$\mathrm{ABI}$, ankle-brachial index; $\mathrm{AD}$, aortic dissection; $\mathrm{ART}$, assisted reproductive technology; BMI, body mass index; $\mathrm{BP}$, blood pressure; CRP, C-reactive protein; CVD, cardiovascular disease; DBP, diastolic blood pressure; eGFR, estimated glomerular filtration rate; GGT, gamma-glutamyl transferase; HbA1c, hemoglobin A1c; HDL, high-density lipoprotein; LDL, low-density lipoprotein; MI, myocardial infarction; NHANES, National Health and Nutrition Examination Survey; OCs, organic compound concentrations; OR, odds ratio; PC, principal component; $\mathrm{PCB}$, polychlorinated biphenyls; PIP, posterior inclusion probability; POP, persistent organic pollutant; PP, pulse pressure; SBP, systolic blood pressure; TC, total cholesterol; TG, triglyceride; TNF, tumor necrosis factor; WBC, white blood cells. * Studies that detected potential interactions within a mixture. " We had to use broader terms, such as "USA" and "Europe", for some studies because these were conducted across all the USA states or European countries. ${ }^{b}$ For the case-control studies, number of cases/number of controls. 


\section{Results}

We identified 1673 studies with MEDLINE (PubMed), 1743 with Scopus, and 1433 with Web of Science (Figure 1). Of these, a total of 3511 citations reflected unique studies. After removing studies that were not peer reviewed, such as book chapters and conference proceedings, 3222 studies were retained for title/abstract screening. The PECO criteria were used to guide title and abstract screening, and 3192 studies were excluded, leaving 30 for full text screening. Of these, three studies were excluded because the metal exposures were not measured at the individual level $[35,36]$ or definitions for the cardiovascular risk factors or outcomes were not consistent with the other studies included [37]; two additional studies $[38,39]$ were identified via manual search or reference lists of the included studies.

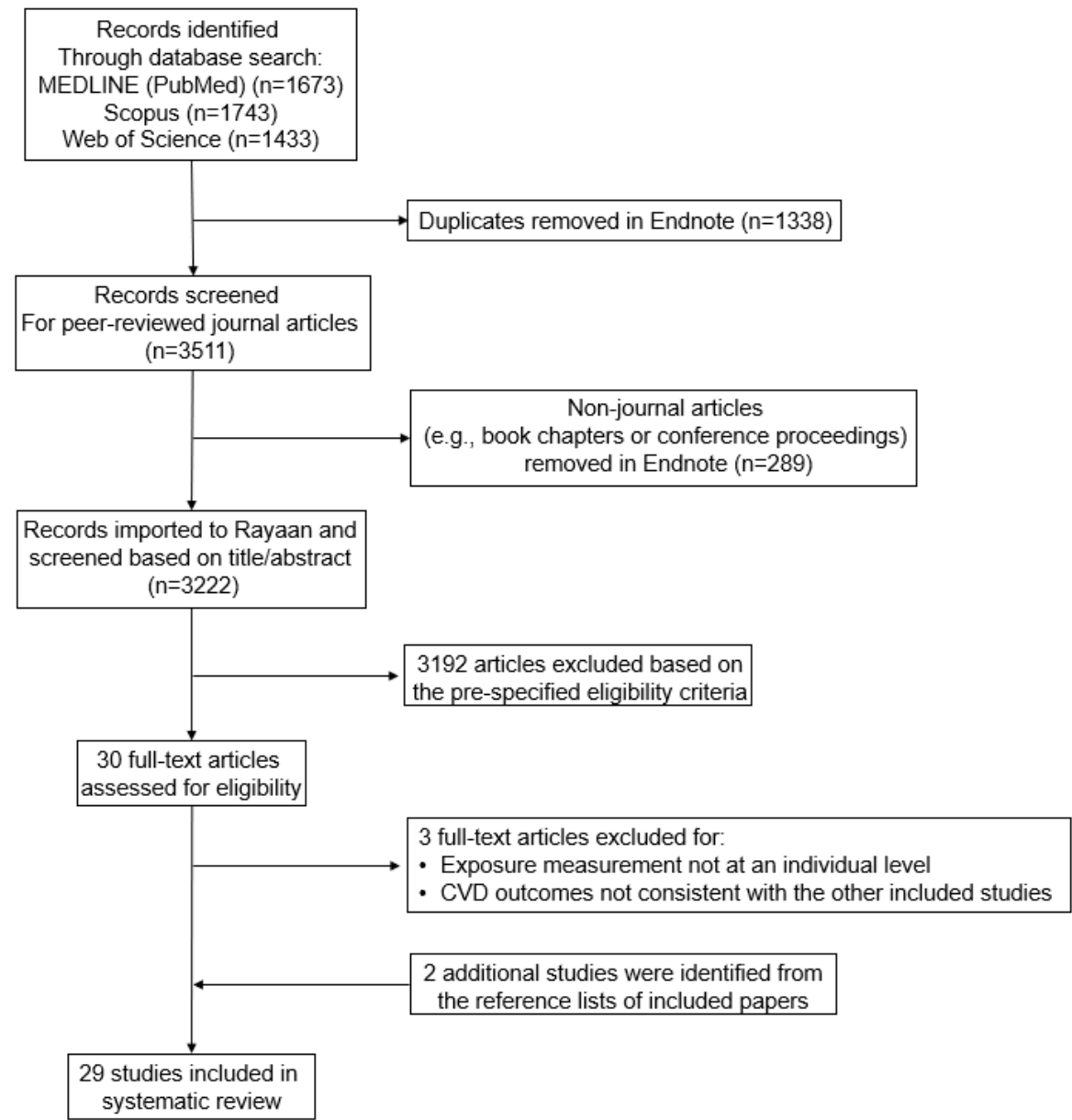

Figure 1. Flow chart of literature review and study selection for papers published between 1998 through 1 October 2021.

We also found two potentially eligible studies but did not include them in this review because mixed-effects regression models, not multipollutant approaches considered in this review, were used among the study population stratified by baseline metal concentrations to estimate joint associations of metal mixtures with blood and pulse pressure trajectories [40] or the clusters identified from a multi-pollutant approach were not linked to CVD-related outcomes [41]. Twenty-nine studies were selected for further review, which were all written in English.

Of the 29 studies included in our review, most $(n=26)$ were published between 2019 and 2021, with the number of studies investigating the association of metal mixtures exposure with CVD risk factors and outcomes doubling in 2021 (Figure 2). Ten studies were conducted in the USA, followed by 9 in China (Table 2). While 6 out of the 10 USA studies were based on NHANES data [42-47], none were excluded as they focused on unique 
combinations of metal components, evaluated different CVD-related outcomes, or used different mixtures analysis methods. Sample sizes ranged between 127 (a panel study of adults in China [48]) to 10,818 (a cross-sectional study conducted in the USA NHANES [43]).

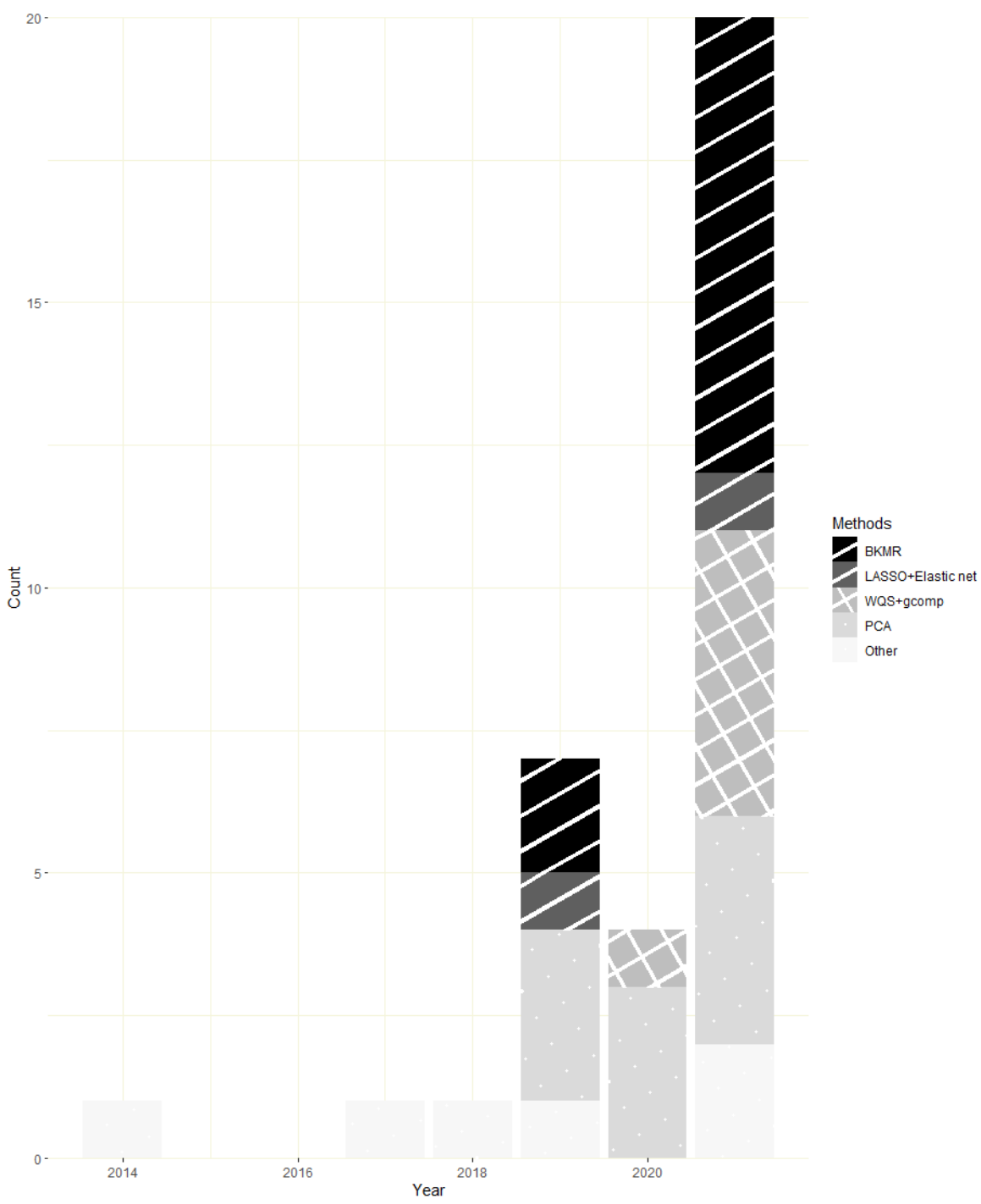

Figure 2. Number of included studies published by year and multi-pollutant approach. Note: Some studies included multiple cardiovascular disease (CVD) related outcomes or mixture analysis methods.

The majority of studies were either cross-sectional in design $(n=13)[39,42-47,49-54]$ or prospective cohort studies $(n=10)$ [38,55-63]. Cardiovascular outcomes and risk factors were categorized into four groups: (i) blood pressure (BP) and hypertension, (ii) preeclampsia, (iii) dyslipidemia and serum lipid markers, and (iv) clinical CVD outcomes, including stroke, coronary heart disease (CHD), and myocardial infarction (MI). Blood pressure and hypertension were the most commonly studied outcomes $(n=15)[38,39,42,44-47,49-51,55-59]$, followed by dyslipidemia and serum or blood lipid markers $(n=5)[43,52,53,55,60]$, and preeclampsia $(n=3)[61,62,64]$. Other CVD outcomes were investigated less frequently $[48,54,55,63,65-68]$.

The frequency by which specific metals have been evaluated is shown separately by CVD risk factors or outcomes in Figure 3. Metal components from each included study were pooled out, rearranged, and counted by the four CVD-related outcomes. Among the included studies, a total of 31 different metals were studied in relation to CVD risk factors or outcomes. The most commonly studied metal was $\mathrm{Cd}$, followed by $\mathrm{Pb}$, mercury $(\mathrm{Hg})$, and 
As. While most of the retrieved studies included both toxic metals and essential elements in their mixtures, five studies $[43,44,46,49,64]$ only included toxic metals in their analysis, with one study [67] focusing on essential elements only. Overall, the results were inconsistent within and across the different endpoints. Possible reasons for these discrepancies include differences in population demographics, exposure patterns and levels, mixture components, study designs, biospecimens measured, the timing of exposure and outcome assessment, population-specific unmeasured confounding, CVD outcome definitions, and the statistical approaches used [69].

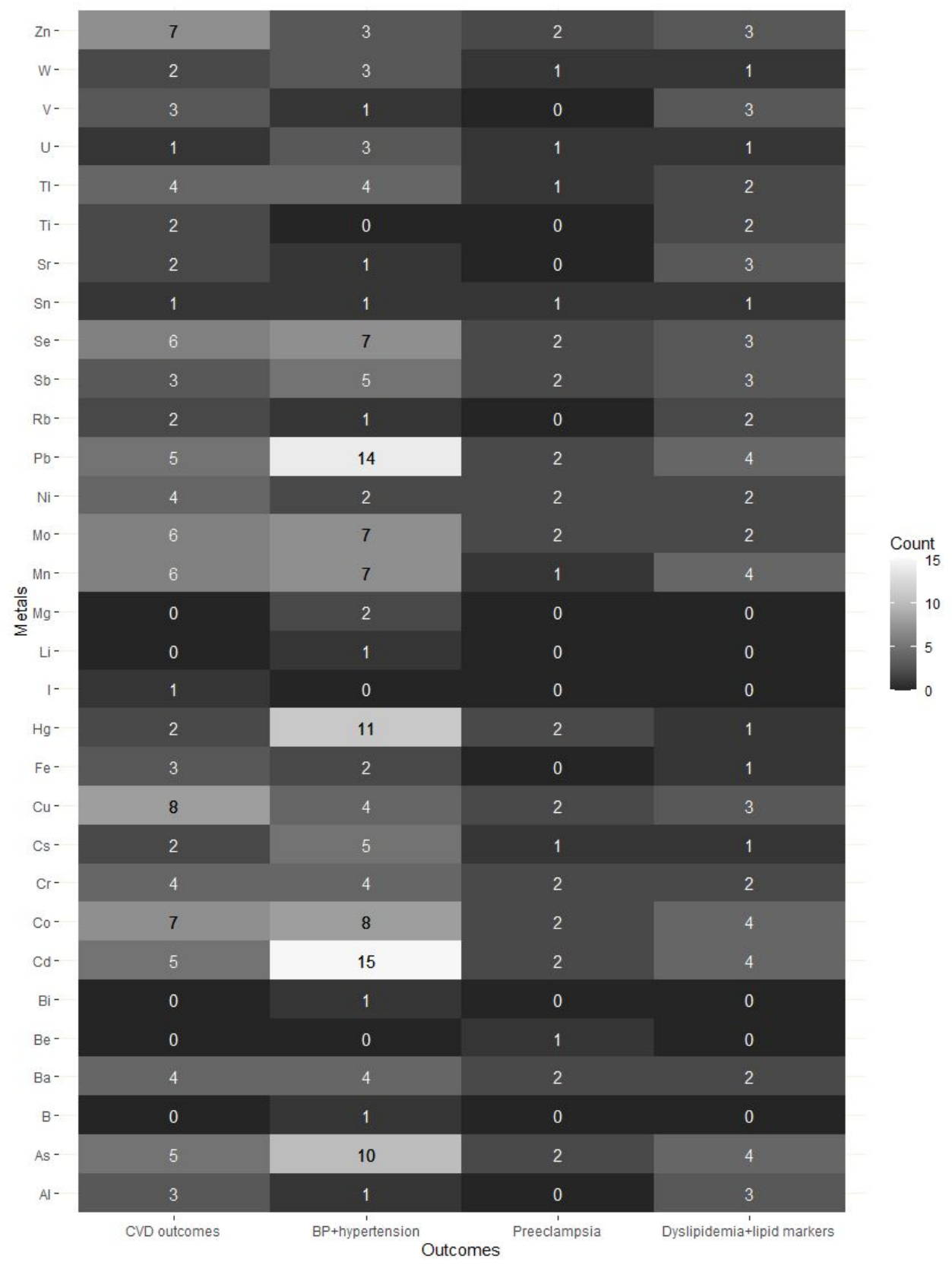

Figure 3. Heat map of metals and cardiovascular disease (CVD) risk factors or outcomes in the included studies. Numbers represent the number of reviewed publications corresponding to each metal (columns) and CVD risk factors or outcomes (rows). Note: The sum of the numbers presented here exceed the total number of selected studies $(n=29)$, as each study included a minimum of three metals within their mixtures. Some studies also evaluated more than one CVD-related outcome or risk factor, including stroke, coronary heart disease, and myocardial infarction; blood pressure; diagnosis of hypertension, preeclampsia, and dyslipidemia; and levels of lipid serum or blood lipid markers. 
Blood was most commonly used for metals assessment $(n=15)$, followed by both blood and urine $(n=7)$, urine $(n=6)$, and toenail clippings $(n=1)$. The majority of studies quantified metal concentrations using inductively coupled plasma-mass spectrometry (ICP-MS), although one study [59] used inductively coupled plasma optical emission spectrometry and one study [55] used inductively coupled plasma triple quadrupole mass spectrometry (ICP-QQQ). Metal concentrations for each of the 29 studies are shown in Table S2. The most frequently employed mixture analysis methods included BKMR, WQSR, and principal component analysis (PCA). Most of the studies adjusted or matched for key confounding factors, such as age, sex and smoking status, in their analyses [17].

\subsection{Blood Pressure and Hypertension}

A total of 15 studies evaluated metal mixtures in relation to blood pressure and risk of hypertension $[38,39,42,44-47,49-51,55-59]$. Almost half of these studies $(n=7)$ were conducted in the USA $[42,44-47,50,58]$. The remaining studies were conducted in Bangladesh [57], Canada [51], China [59], Greece [56], Mexico [55], South Korea [49], Spain [39], or across Europe [38].

Almost half $(n=7)$ of these studies focused on child or adolescent populations, of which four studies focused on metal mixture exposures during the prenatal period. Sample sizes ranged from 133 to 10,566 participants. Most of the studies were cross-sectional in design [39,42,44-47,49-51], and the remaining studies were cohort studies [38,55-59]. Metal concentrations were most frequently measured in blood $(n=6)$, followed by both blood and urine $(n=5)$, urine $(n=3)$, and toenail clippings $(n=1)$. Procedures for blood pressure measurement and hypertension definitions were consistent across the included studies. However, diverse multipollutant approaches were employed, including PCA, BKMR, WQSR, quantile-based g-computation, k-medoids, regression tree, environmental risk score (ERS), and deletion-substitution-addition (DSA) algorithm.

Six studies meeting our inclusion criteria, all conducted in adults, have investigated impacts of metal mixtures on hypertension. Of these, only one prospective study by Zhong et al. [59] has investigated metal mixture impacts on hypertension. This study was conducted in the Wanjiang Cohort, which follows adults living along the Yangtze River in China. A large panel of metals, measured in urine (aluminum [Al], As, boron [B], barium [Ba], bismuth [Bi], Cd, cobalt [Co], $\mathrm{Cr}$, copper [Cu], iron [Fe], Hg, lithium [Li], magnesium [Mg], manganese [Mn], molybdenum [Mo], nickel [Ni], Pb, rubidium [Rb], selenium [Se], strontium [Sr], titanium [Ti], and zinc [Zn]), were examined in relation to hypertension using a 2-stage approach. First, single-metal analyses were conducted using traditional linear regression. Hierarchical BKMR was then used to simultaneously evaluate metals that were found to be associated significantly with hypertension in step 1 as a mixture. Metals were grouped based on their pairwise correlations and knowledge of common exposure sources. BKMR identified a positive association between $\mathrm{Cd}$ and hypertension, and similar trends were observed for Mo and Zn. A cumulative increase in all metals was also associated with greater odds of hypertension. Additionally, a potential interaction between $\mathrm{Cd}$ and $\mathrm{Zn}$ was detected in relation to risk of hypertension. Park et al. [42] conducted a cross-sectional analysis of metal mixtures, measured in both blood and urine, and hypertension among adults in the USA NHANES. An ERS was constructed as a summary measure to investigate the multipollutant impact of a large panel of metals (antimony [Sb], As, $\mathrm{Ba}, \mathrm{Cd}, \mathrm{Co}$, cesium [Cs], $\mathrm{Mo}, \mathrm{Hg}, \mathrm{Pb}$, thallium [Tl], tungsten [W], and uranium [U] in urine; $\mathrm{Cd}, \mathrm{Hg}$, and $\mathrm{Pb}$ in whole blood; and arsenobetaine, monomethyl arsenic, and dimethyl arsenic in urine) on gamma-glutamyl transferase (GGT) using adaptive elastic-net with main effects and pairwise interactions (AENET-I), Bayesian additive regression tree (BART), BKMR, and Super Learner. In the second step, associations between the GGTERS and cardiovascular endpoints, including hypertension, were evaluated. The authors concluded that the ERS based on AENET performed better than the other approaches in terms of prediction performance. The ERS included Cd (urine), dimethylarsonic acid, monomethylarsonic acid, Co, and Ba as significant predictors of GGT, and all ERS showed 
significant associations with risk of hypertension, with a one standard deviation (SD) increase in ERS from AENET-I being associated with a 1.26 (95\% CI: 1.15-1.38) higher odds of hypertension. Wang et al. [45] similarly examined the association between exposure to the same set of metals that Park et al. evaluated [42] and hypertension among the USA NHANES participants using a 2-stage approach. In step 1, AENET was used to identify which elements from the mixture [42] were associated with waist circumference. While both studies constructed ERS using AENET, Wang et al. additionally included squared terms and pairwise interactions in their model. In step 2, an ERS was constructed from the metals selected in step 1 , including $\mathrm{Pb}, \mathrm{Cd}, \mathrm{Hg}, \mathrm{Ba}$, and $\mathrm{Tl}$, which was then examined in relation to hypertension. The 90th versus 10th percentile of the ERS was associated with a 1.55 (95\% CI: 1.28-1.88) higher odds of hypertension. Another cross-sectional study by $\mathrm{Xu}$ et al. [50] utilized data from the Gulf Long-Term Follow-Up Study in the USA, a cohort of individuals who participated in cleanup efforts for the Deepwater Horizon oil spill and individuals who completed safety trainings but were not hired. Whole blood measures of multiple metals $(\mathrm{Cd}, \mathrm{Hg}, \mathrm{Mn}, \mathrm{Pb}$, and $\mathrm{Se})$ were investigated simultaneously in relation to hypertension using quantile-based g-computation, but results were null (OR: $0.96,95 \%$ CI: $0.73-1.27$ for a quartile increase in the metal mixture). In a cross-sectional analysis of adults in the Korean NHANES, Kim and Park [49] examined the combined impact of three metals $(\mathrm{Cd}, \mathrm{Hg}$, and $\mathrm{Pb})$ measured in whole blood on hypertension and prehypertension using WQSR. A quartile increase in the WQS index was associated with greater odds of hypertension (odds ratio [OR]:1.29, 95\% confidence interval [CI]: 1.19-1.40), with $\mathrm{Pb}$ contributing the most to this association.

Of the six studies which evaluated metals in relation to risk of hypertension, only one by Zuk et al. [51] considered other chemicals as part of the mixture. The authors used PCA to investigate how exposure to multiple classes of environmental contaminants were associated with risk of hypertension among indigenous adults residing in Canada. The environmental chemicals mainly consisted of persistent organic chemicals (nine polychlorinated biphenyls [PCBs] and seven organochlorine pesticides or metabolites), along with four metals $(\mathrm{Cd}, \mathrm{Hg}, \mathrm{Pb}$, and $\mathrm{Se})$ measured in blood. The top two principal components (PCs) had high loadings for all PCBs, other organic compound concentrations (OCs), and $\mathrm{Hg}$ (PC-1), or Pb (PC-2). In the modified Poisson regression model adjusting for these two PCs as independent predictors for risk of hypertension, PC-1 was associated with higher risk of hypertension (adjusted prevalence ratios [aPR]: 1.08; 95\% CI: 1.003-1.172).

Two studies examined metal mixture impacts on blood pressure (BP) in adults. Everson et al. [47] used regression trees to identify predictors for systolic blood pressure (SBP) and diastolic blood pressure (DBP) from nine metals (Ba, Cd, Co, Cs, Mo, Sb, Tl, $\mathrm{W}$ measured in urine, $\mathrm{Pb}$ measured in whole blood) among those below the age of 60 in the USA NHANES. They found that higher concentrations of $\mathrm{Sb}, \mathrm{Cd}, \mathrm{W}$, and $\mathrm{Pb}$ predicted higher SBP, while Cs and Mo predicted lower SBP and DBP. Predictors varied by race/ethnicity, as $\mathrm{Cd}$ was the main predictor for SBP among non-Hispanic black adults, whereas the combination of high $\mathrm{W}$ and high $\mathrm{Sb}$ was the main predictors for SBP among non-Hispanic white adults. Similarly, Yao et al. [44] assessed metal mixtures impacts on BP in the USA NHANES using a two-step approach. In the first step, they applied k-medoids clustering to categorize the study population into two subgroups based on either urine levels of $\mathrm{Pb}, \mathrm{Cd}$, and total $\mathrm{As}$, or blood levels of $\mathrm{Pb}, \mathrm{Cd}$, and $\mathrm{Hg}$. Secondly, linear regression models were used to examine the association between the exposure subgroups and BP. The "high-exposure" group based on blood levels was significantly associated with increased SBP and DBP compared with the "low-exposure" group, and the "high-exposure" group based on urine levels was significantly associated with increased DBP, but not SBP.

Four prospective cohort studies investigated the impacts of prenatal exposure to metal mixtures on childhood BP. Zhang et al. [58] examined the relationship between exposure to five metals $(\mathrm{Pb}, \mathrm{Hg}, \mathrm{Cd}, \mathrm{Se}$, and $\mathrm{Mn}$ in maternal whole blood samples collected 24 to $72 \mathrm{~h}$ after delivery) and childhood BP between 3 and 15 years of age from the Boston Birth Cohort. Overall, metal mixtures were not associated with BP. BKMR with hierarchical 
variable selection identified essential elements as having stronger associations with SBP than toxic metals. Among essential elements and toxic metals, Se and $\mathrm{Pb}$, respectively, had the largest conditional posterior inclusion probabilities (PIPs), which quantify the relative importance of each component within the mixture. BKMR also identified a potential interaction between $\mathrm{Mn}$ and $\mathrm{Cd}$, indicating that the inverse association between $\mathrm{Mn}$ and childhood SBP was strong at higher concentrations of $\mathrm{Cd}$. Howe et al. [56] assessed the association between prenatal exposure to metal mixtures $(\mathrm{Mg}, \mathrm{Co}, \mathrm{Se}, \mathrm{Mo}, \mathrm{As}, \mathrm{Cd}, \mathrm{Sb}$, and $\mathrm{Pb}$ measured in maternal urine samples collected in early pregnancy) and elevated BP at 11 years of age in the Rhea cohort in Greece using BKMR. They also used Bayesian Varying coefficient kernel machine regression (BVCKMR) to assess the impact of this mixture on BP trajectories from 4-11 years of age. A J-shaped association was found between Mo and Co exposure with both SBP and DBP at baseline (age 4). Cd was inversely associated with DBP but not with SBP at baseline. From ages 4 to 11, however, Mo was associated with lower per-year increases in DBP, and Co was associated with lower per-year increases in both SBP and DBP. Mg was not associated with BP at baseline but was associated with higher per-year increases in both SBP and DBP from ages 4 to 11 . Mo and Pb showed a J-shaped association with BP at age 11, and a possible synergistic interaction was identified between $\mathrm{Mo}$ and $\mathrm{Pb}$ and $\mathrm{BP}$ at ages 4 and 11. Kupsco et al. [55] evaluated the association between prenatal exposure to eleven metals ( $\mathrm{As}, \mathrm{Cd}, \mathrm{Co}, \mathrm{Cr}, \mathrm{Cs}, \mathrm{Cu}, \mathrm{Mn}, \mathrm{Pb}, \mathrm{Sb}$, Se, and $\mathrm{Zn}$ measured in maternal whole blood samples collected during the second trimester) and BP among children 4-6 years old in the Mexico Programming Research in Obesity, Growth Environment and Social Stress (PROGRESS) birth study. BKMR indicated that the joint and individual effect of metals on BP were close to null. Warembourg et al. [38] conducted an exposome-wide association study (ExWAS) using DSA to study associations between the prenatal and postnatal exposomes and childhood BP at 6-11 years of age using pooled data from 6 longitudinal-based European birth cohorts. The prenatal exposomes included 10 metals As, Cd, Co, Cs, Cu, Hg, Mn, Mo, Pb, and Tl measured in maternal whole blood samples collected during pregnancy. The postnatal exposomes included the same 10 metals measured in child blood samples collected at 6-11 years of age. The prenatal and postnatal exposomes were investigated separately in relation to child BP. Postnatal $\mathrm{Cu}$ was associated with increased DBP at 6-11 years of age, but none of the prenatal metal exposures were selected as important predictors of child BP.

Desai et al. [46] used BKMR to evaluate associations between metal mixtures ( $\mathrm{Pb}$ and $\mathrm{Hg}$ in whole blood; $\mathrm{Cd}$ and total As in urine) on BP among children and adolescents between 8 and 17 years of age in the USA NHANES. BKMR identified inverse associations between low-level $\mathrm{Pb}, \mathrm{Hg}$, As, and $\mathrm{Cd}$ and DBP. A suggestive inverse association was also identified between $\mathrm{Cd}$ and both SBP and DBP. Notably, urinary Cd concentrations (median: $0.06 \mu \mathrm{g} / \mathrm{g}$ creatinine) among this NHANES sample of children and adolescents were low, with $37 \%$ of $\mathrm{Cd}$ measures in this population falling below the LOD (limit of detection). Castiello et al. [39] used PCA to determine the impacts of metal mixtures (As, Cd, $\mathrm{Hg}, \mathrm{Ni}$, $\mathrm{Pb}, \mathrm{Mn}$, and $\mathrm{Cr}$ in urine) on BP among Spanish male adolescents 15-17 years of age. PC-1 had high loadings from Ni, Cr, and Mn; PC-2 had high loadings for As and Hg; and PC-3 had high loadings for $\mathrm{Cd}$ and $\mathrm{Pb}$. A positive association was found between $\mathrm{PC}-2$ and SBP. Shih et al. [57] used three mixture approaches (BKMR, PCA, and WQS) to evaluate the association of seventeen metals ( $\mathrm{Al}, \mathrm{As}, \mathrm{Cd}, \mathrm{Cr}, \mathrm{Co}, \mathrm{Cu}, \mathrm{Fe}, \mathrm{Pb}, \mathrm{Mn}, \mathrm{Hg}, \mathrm{Mo}, \mathrm{Ni}, \mathrm{Se}$, tin [Sn], U, vanadium [V], and Zn measured in child's toenail) with BP at 5-7 years of age in Bangladesh. BKMR identified an overall negative association between the metal mixture and SBP. Of all metals, Sn had the largest PIP for SBP and Se, Mo and $\mathrm{Hg}$ had the highest PIPs for DBP. Cr was positively associated with SBP and DBP among boys but not girls, whereas $\mathrm{Cu}$ was negatively associated with DBP among girls but not boys. Most relationships were linear, except for the associations of Sn with SBP, and Cu and Se with DBP in girls. The WQS positive index was associated with increased DBP in the whole study population ( $\beta$ coefficient: 1.07, 95\% CI: $0.09-2.05)$ and also among boys ( $\beta$ coefficient: 1.68, 95\% CI: 0.21-3.14). Se, Ni and Zn contributed the most to the positive index for DBP 
in the overall population while $\mathrm{Mo}, \mathrm{Se}, \mathrm{Ni}, \mathrm{Cd}, \mathrm{Pb}$ and $\mathrm{As}$ contributed most to the index among boys. The authors also used PCA, focusing on relationships between the top three PCs and BP. Among boys, PC-2 (highly loaded with $\mathrm{Cu}, \mathrm{Zn}, \mathrm{Se}, \mathrm{Cd}$ and $\mathrm{Pb}$ ) was associated with higher DBP, and PC-3 (highly loaded with $\mathrm{Hg}$ ) was associated with lower DBP. Among girls, Sn, Se and Zn had higher loadings for PC3, which was associated with a lower SBP.

\subsection{Preeclampsia}

Three studies based in the USA [61,62] and China [64] investigated metal mixture impacts on risk of preeclampsia or changes in blood pressure during pregnancy $[61,62,64]$. The sample sizes ranged from 383 to 1688. Of these, two studies were prospective cohort studies [61,62] and the remaining study was case-controlled in design [64]. No metal was included in all three studies, but $\mathrm{As}, \mathrm{Cd}, \mathrm{Co}, \mathrm{Cr}, \mathrm{Hg}, \mathrm{Ni}, \mathrm{Pb}$, and $\mathrm{Sb}$ were each included in two of the three studies. Liu et al. [62] assessed the association between exposure to 8 metals (Ba, Cs, Sb, Co, Cu, Mo, Se, and $\mathrm{Zn}$ ) measured in first trimester blood samples and $\mathrm{BP}$ at baseline as well as BP changes during pregnancy. Study participants were drawn from the Eunice Kennedy Shriver National Institute of Child Health and Human Development Fetal Growth Studies-Singleton cohort in the USA. Using BKMR, a joint association was observed between the metal mixture and both SBP and DBP at baseline. Individual metals, accounting for the rest of the mixture, were also evaluated; each interquartile range (IQR) increase in $\mathrm{Cu}$ was associated with a $0.67 \mathrm{mmHg}(95 \% \mathrm{CI}$ : 0.02-1.32) higher SBP and a $0.60 \mathrm{mmHg}$ (95\% CI: 0.08-1.12) higher DBP at baseline. Similarly, each IQR increment in Se was associated with a $0.67 \mathrm{mmHg}$ higher SBP (95\% CI: 0.05-1.29). The study by Bommarito et al. [61] included pregnant women enrolled in the LIFECODES birth cohort in Boston who were originally selected for a case-control study of preterm birth; inverse probability weighting was used to account for the disproportionate number of preterm births in their sample. Metal mixture impacts on preeclampsia were examined using PCA, focusing on 11 metals measured in third trimester maternal urine samples that were above detection for $>30 \%$ of participants: As, beryllium [Be], Cd, Cu, Hg, Mn, Ni, $\mathrm{Pb}, \mathrm{Se}, \mathrm{Sn}, \mathrm{Tl}$, and Zn. The top three metal PCs were not significantly associated with preeclampsia, and no significant interactions were identified between the PCs. However, individuals with higher scores for PC-2 (characterized by high loadings for $\mathrm{Cd}, \mathrm{Mn}$, and $\mathrm{Pb}$ ) who had scores below the median for PC-1 (characterized by high loadings for $\mathrm{Cu}$, Se, and $\mathrm{Zn}$ ) had an increased risk of developing preeclampsia. Wang et al. [64] evaluated associations between eight metals $(\mathrm{Cr}, \mathrm{Co}, \mathrm{Pb}, \mathrm{Hg}, \mathrm{Sb}, \mathrm{Cd}, \mathrm{As}$, and $\mathrm{Ni}$ ), measured in maternal whole blood collected at delivery, and preeclampsia using two mixture modeling approaches: WQSR and PCA. With WQSR, they found that a tertile increase in the weighted sum of metals was associated with higher odds of developing preeclampsia (OR: 1.68, 95\% CI: 1.20-3.33), with $\mathrm{Cr}$ contributing the most to this association. In the PCA analysis, individuals with scores in the highest tertile for PC-2 (characterized by high loadings for $\mathrm{Cr}$ and As) had an increased odds of preeclampsia (OR: 1.59, 95\% CI: 1.10-2.31) compared with individuals with scores in the lowest tertile. Furthermore, individuals with scores in the highest and middle tertiles for PC-3 (characterized by high loadings for $\mathrm{Pb}$ and $\mathrm{Hg}$ ) had an increased odds of early onset preeclampsia (gestational age $<34$ weeks) compared to individuals with scores in the lowest tertile (OR: 2.19, 95\% CI: 1.34-3.60 and OR: 2.48, 95\% CI: $1.45-4.25$ comparing the middle and high tertiles with the lowest tertile, respectively). In stratified analyses, the positive association between PC-2 and preeclampsia was stronger among women who were overweight or obese. Both Bommarito et al. and Wang et al. used the same definition of preeclampsia.

\subsection{Dyslipidemia and Lipid Markers}

Five peer-reviewed papers which met our inclusion criteria examined associations between metal mixture exposures and risk of dyslipidemia $[52,60]$ or lipid markers, such as triglyceride (TG), total cholesterol (TC), high-density lipoprotein cholesterol (HDL-C), low-density lipoprotein cholesterol (LDL-C), and leptin levels $[43,53,55,60]$. These studies 
were conducted in China [52,53,60], the USA [43], and Mexico [55] with sample sizes ranging from 461 to 10,818 participants. Two of these were cohort studies, and three were cross-sectional in design. The investigators used a range of methods to evaluate the impacts of metal mixtures on dyslipidemia, including PCA, ERS, WQSR, and BKMR.

Jiang et al. [60] and Zhu et al. [52] investigated the associations of exposure to multiple metals with risk of dyslipidemia. Both studies: (i) used the same definition of dyslipidemia, (ii) measured metal concentrations in blood samples, (iii) employed a combination of PCA and logistic regression, and (iv) adjusted for a similar set of covariates. However, the two studies differed by participant age distribution and study design. Jiang et al. [60] measured a large panel of metals ( $\mathrm{Al}, \mathrm{Sb}, \mathrm{As}, \mathrm{Ba}, \mathrm{Co}, \mathrm{Cu}, \mathrm{Pb}, \mathrm{Mn}, \mathrm{Mo}, \mathrm{Ni}, \mathrm{Rb}, \mathrm{Se}, \mathrm{Sr}, \mathrm{Tl}, \mathrm{Ti}, \mathrm{V}$, and $\mathrm{Zn}$ ) in plasma among participants drawn from prior studies nested within the Dongfeng-Tongji cohort in China. Participants were prospectively followed for an average of 5 years to ascertain incident dyslipidemia using plasma measurements of TC, TG, HDL-C, and LDL-C. PCA results suggested that individuals with scores in the highest quartile of PC-1 (which had high loadings for plasma $\mathrm{Al}, \mathrm{As}, \mathrm{Ba}, \mathrm{Pb}, \mathrm{V}$, and $\mathrm{Zn}$ ) had a $40 \%$ increase in risk of dyslipidemia (OR: 1.40; 95\% CI: 1.07-1.84) compared with individuals with scores in the lowest quartile. No significant associations were identified for the other four PCs evaluated and dyslipidemia. Zhu et al. used a cross-sectional design and focused on adults $>60$ years old in China [52]. They used PCA to reduce the dimensionality of seven metals ( $\mathrm{Sr}, \mathrm{Cd}$, $\mathrm{Pb}, \mathrm{V}, \mathrm{Al}, \mathrm{Co}$, and $\mathrm{Mn}$ ) measured in blood. Combined exposure to $\mathrm{Al}, \mathrm{Co}$, and $\mathrm{V}$ was associated with reduced risk for dyslipidemia, whereas $\mathrm{Cd}$, $\mathrm{Sr}$, and $\mathrm{Pb}$ were associated with an increased risk.

Jiang et al. [60], Li et al. [53], and Park et al. [43] reported relationships between exposure to metal mixtures and concentrations of lipid markers in adult populations. Metals measured in at least two out of these three studies included $\mathrm{Al}, \mathrm{Ba}, \mathrm{Cd}, \mathrm{Co}, \mathrm{Cu}, \mathrm{Mn}$, $\mathrm{Mo}, \mathrm{Ni}, \mathrm{Pb}, \mathrm{Rb}, \mathrm{Sb}, \mathrm{Se}, \mathrm{Sr}, \mathrm{Ti}, \mathrm{Tl}, \mathrm{V}$, and $\mathrm{Zn}$; As was evaluated in all three studies. Utilizing a combination of PCA and logistic regression, Jiang et al. [60] reported positive associations between $\mathrm{PC}-1$ (characterized by high loadings for $\mathrm{Al}, \mathrm{As}, \mathrm{Ba}, \mathrm{Pb}, \mathrm{V}$, and $\mathrm{Zn}$ ) and risk of low HDL-C and high LDL-C, and between PC-3 (characterized by high loadings for $\mathrm{Cu}$, $\mathrm{Rb}$, and Se) and risk of high TC and low HDL-C. Li et al. [53] conducted a cross-sectional study among healthy adults living in the Shimen and Huayuan Counties of the Hunan Province in China to investigate the influence of toxic metals, measured in urine, and micronutrient metals, measured in plasma, on blood lipids (TG, TC, HDL-C and LDL-C) using a combination of WQSR and BKMR-based approaches. Among participants in the Huayuan area, a grouped WQSR analysis identified positive associations between essential metals ( $\mathrm{Co}, \mathrm{Cr}, \mathrm{Cu}, \mathrm{Fe}, \mathrm{Mn}, \mathrm{Mo}, \mathrm{Ni}, \mathrm{Se}, \mathrm{Sn}, \mathrm{Sr}, \mathrm{V}$, and $\mathrm{Zn}$ ) and higher concentrations of $\mathrm{TG}, \mathrm{TC}$, and LDL-C and an inverse association between toxic metals ( $\mathrm{Al}, \mathrm{As}, \mathrm{Ba}, \mathrm{Cd}, \mathrm{Rb}$, $\mathrm{Sb}, \mathrm{Ti}, \mathrm{Tl}, \mathrm{U}$, and $\mathrm{W}$ ) and TG and TC. In contrast, BKMR models did not provide strong evidence of joint effects. However, the BKMR analysis identified associations for both $\mathrm{Ti}$ and $\mathrm{Zn}$ with TG levels in both study sites; associations for other metals varied by site. Nonlinear associations with total cholesterol and LDL-C were also observed for certain metals, including an inverse U-shaped relation between plasma Fe and LDL-C in the Huayuan area. Moreover, a possible interaction between $\mathrm{Zn}$ and $\mathrm{Cd}$ was observed in relation to LDL-C in the Huayuan area. Using data from the USA NHANES, Park et al. [43] developed an ERS incorporating measurements of 134 environmental toxicants, including blood concentrations of $\mathrm{Pb}, \mathrm{Cd}$, and total $\mathrm{Hg}$ and urinary concentrations of $\mathrm{Cd}, \mathrm{Hg}$, and both total and speciated As. The ERS was constructed as a weighted sum of the exposures that were selected using regression analysis, followed by logistic regression models with dichotomized each blood lipid measure (TC, HDL, LDL, and TG). Among the pollutants identified as important predictors of blood lipids, urinary $\mathrm{Sb}$ was associated with elevated $\mathrm{HDL}$ ( $>40 \mathrm{mg} / \mathrm{dL}$ for men and $>50 \mathrm{mg} / \mathrm{dL}$ for women), whereas the associations for urinary $\mathrm{Cd}$ and blood $\mathrm{Pb}$ were not significant. In contrast, blood $\mathrm{Pb}$ showed a significant positive association with the odds of elevated LDL ( $>130 \mathrm{mg} / \mathrm{dL})$. Although urinary Cd was also 
positively associated with the odds of elevated LDL, the association was weak and was not statistically significant.

Of the selected studies, Kupsco et al. [55] was the only study that investigated the association between prenatal exposure to metal mixtures and the concentrations of lipid markers among children. While a higher level of Se was associated with lower TG, higher concentrations of $\mathrm{Sb}$ and As were associated with lower leptin levels. Kupsco et al. did not identify evidence for interactions within the metal mixture or non-linear relationships.

\subsection{Additional CVD Outcomes}

We identified eight studies which evaluated the relationship between exposure to metal mixtures and other CVD outcomes [48,54,55,63,65-68]. Diverse CVD outcomes were examined across studies, including stroke [63,65-67], incident CHD [63], incident MI [67], carotid intima-media thickness [54], aortic dissection (AD) [68], arterial stiffness of peripheral arteries [48], and cardiometabolic component scores [55]. Half of these studies $(n=4)$ were conducted in China $[48,65,66,68]$, with the remaining studies performed in Canada [54], Germany [67], Mexico [55], and Spain [63]. A range of study designs, including case-control $(n=3)$, cohort $(n=2)$, case-cohort $(n=1)$, cross-sectional $(n=1)$, and panel studies $(n=1)$ were used for these investigations. Sample sizes ranged between 127 to 2554 participants. Most studies $(n=6)$ measured metal concentrations in blood, while two measured metal concentrations in urine. A wide range of multipollutant approaches were employed across these studies, including PCA, BKMR, WQSR, Elastic net, and least absolute shrinkage and selection operator (LASSO).

Cabral et al. [67], Domingo-Relloso et al. [63], Wen et al. [65], and Xiao et al. [66] reported the associations between metal mixture exposures and stroke. Across these four studies, $\mathrm{Cu}$ and $\mathrm{Zn}$ were evaluated most commonly, followed by $\mathrm{Co}, \mathrm{Mn}, \mathrm{Mo}$, and Se. Findings from Cabral et al. [67] employed PCA to assess risk of incident CVD outcomes (MI and stroke) by co-exposure to six essential elements ( $\mathrm{Mn}, \mathrm{Fe}, \mathrm{Cu}, \mathrm{Zn}$, iodine [I], and $\mathrm{Se}$ ) measured in blood among 2087 adults who participated in the European Prospective Investigation into Cancer and Nutrition (EPIC)-Potsdam cohort study. PC-1 was characterized by high loadings for $\mathrm{Mn}, \mathrm{Fe}$, and $\mathrm{Zn}$, whereas PC-2 was characterized by high loadings for $\mathrm{Cu}$, I, and Se. PC-2 was associated with an increased risk of developing incident MI or stroke. Domingo-Relloso et al. [63] utilized data from 1171 adults in the Hortega Follow-Up Study in Spain. A mixture of nine metals ( $\mathrm{Sb}, \mathrm{Ba}, \mathrm{Cd}, \mathrm{Cr}, \mathrm{Co}, \mathrm{Cu}, \mathrm{Mo}, \mathrm{V}$, and $\mathrm{Zn}$ ) measured in urine were evaluated using a probit extension of BKMR; accounting for the mixture, $\mathrm{Cu}, \mathrm{Zn}$, $\mathrm{Sb}, \mathrm{Cd}, \mathrm{Sr}$, and $\mathrm{V}$ were associated with an increased risk of cardiovascular (CHD and stroke) incidence. The study by Wen et al. [65] used a case-control design and included 2554 adults from Shenzhen, China, measuring 11 metals ( $\mathrm{Al}, \mathrm{As}, \mathrm{Cd}, \mathrm{Co}, \mathrm{Cu}, \mathrm{Fe}, \mathrm{Mn}, \mathrm{Mo}, \mathrm{Se}, \mathrm{Tl}$, and $\mathrm{Zn}$ ) in blood. A positive association was identified between the $\mathrm{PC}$ represented by $\mathrm{Al}, \mathrm{Cd}$, and $\mathrm{Mn}$ and risk of first ischemic stroke, whereas an inverse association was identified for the PC with high loadings for Fe and Se. The study by Xiao et al. [66] included a total of 1035 cases and 1035 controls for ischemic stroke and 269 cases and 269 controls for hemorrhagic stroke in the Dongfeng-Tongji cohort in China. Out of 18 metals measured in blood (Al, As, $\mathrm{Ba}, \mathrm{Co}, \mathrm{Cu}, \mathrm{Pb}, \mathrm{Mn}, \mathrm{Hg}, \mathrm{Mo}, \mathrm{Ni}, \mathrm{Rb}, \mathrm{Se}, \mathrm{Sr}, \mathrm{Tl}, \mathrm{Ti}, \mathrm{W}, \mathrm{V}$, and $\mathrm{Zn}$ ), elastic net selected $\mathrm{Cu}, \mathrm{Mo}$, and $\mathrm{Se}$ as metals associated with ischemic stroke and $\mathrm{Rb}$ and $\mathrm{Se}$ as metals associated with hemorrhagic stroke. The regression coefficients for these selected metals from the elastic net model were used as weights and summed to construct predictive plasma metal scores. In adjusted conditional logistic regression model, the calculated predictive plasma metal scores were positively associated with risk of ischemic stroke (OR: 1.37; 95\% CI: 1.20, 1.56) and hemorrhagic stroke (OR: 1.53; 95\% CI: 1.16-2.01).

Other cardiovascular outcomes evaluated included carotid intima-media thickness, AD, arterial stiffness of peripheral arteries, and global metabolic risk score. Liberda et al. [54] examined 43 contaminants, including 10 metals $(\mathrm{As}, \mathrm{Pb}, \mathrm{Cd}, \mathrm{Hg}$, Se, Co, Cu, Mo, Ni, and $\mathrm{Zn}$ ) measured in blood, in relation to carotid intima-media thickness in the Environmentand-Health Study in the Eeyou Istchee territory of Quebec, Canada. Using PCA as their 
multipollutant approach, Of PCs identified, only PC-5, which had a high loading for $\mathrm{Ni}$, was statistically significantly associated with increased carotid intima-media thickness. Liu et al. [68] investigated metal mixture associations with risk of AD, employing WQSR and BKMR as their multipollutant approaches. The study population included a total of 310 adults in Nanjing, China, and concentrations of 10 metals (Mo, Tl, Cu, Cs, Ba, Pb, Cr, $\mathrm{Mn}, \mathrm{Co}$, and $\mathrm{Ni}$ ) were measured in blood. The WQSR analysis indicated an elevated risk of $\mathrm{AD}$ for each quantile increase in the metal mixture (coefficient: 3.49, 95\% CI: 2.25-5.28) and identified $\mathrm{Cu}, \mathrm{Ni}$, and $\mathrm{Mo}$ as the main mixture components contributing to this association. Similarly, the BKMR analysis showed a positive association between the metal mixture and risk of $\mathrm{AD}$, pinpointing $\mathrm{Cu}$ and $\mathrm{Mo}$ as the most influential contributors based on their PIPs. The study by Yang et al. [48] was conducted among 127 adults in Wuhan, China. Out of the 23 metals (Al, Sb, As, Ba, Cd, Cr, Co, Cu, Fe, Pb, Mn, Mo, Ni, Rb, Se, Sr, Tl, Sn, Ti, $\mathrm{W}, \mathrm{U}, \mathrm{V}$, and $\mathrm{Zn}$ ) measured in urine, LASSO identified Ti and Co as important predictors of arterial stiffness of peripheral arteries. Interestingly, the inverse association between $\mathrm{Ti}$ and a measure of arterial stiffness of peripheral arteries was more pronounced among never-smokers. However, a study of 548 participants from a prospective birth cohort in Mexico by Kupsco et al. [55] did not find evidence for a cumulative association between 11 metals (As, Cd, Co, Cr, Cs, Cu, Mn, Pb, Sb, Se, and $\mathrm{Zn}$ ) measured in maternal blood during pregnancy and global metabolic risk score among the offspring.

\subsection{Statistical Methods for Analyzing Effects of Chemical Mixtures}

Across studies, the most frequently used multipollutant approaches included PCA, BKMR, and WQSR. Importantly, the goals and assumptions of these modeling techniques differ (Table S3). PCA is an unsupervised, dimension reduction technique which aims to explain as much of the total variance in the data as possible using the minimum number of components [70]. While PCA is widely employed, it has important limitations, including: (1) generating components that may not be easily interpreted, (2) reliance on the researcher's judgement for deciding the number of components to retain, and (3) the possibility of selecting environmental chemicals that may be irrelevant to the outcomes of interest, as it is an unsupervised approach [71]. In contrast, BKMR is a supervised, semi-parametric method that examines exposure-response relationships using a non-parametric kernel function, while parametrically accounting for covariates. BKMR can evaluate the health impacts of environmental chemicals both individually and jointly, allowing for non-linearity and non-additivity in these associations. BKMR also includes a hierarchical variable selection function, which allows researchers to group chemical mixture components using prior knowledge to determine their group-wise relationships with health outcomes. In addition, PIPs are calculated to estimate the relative importance of each element in the overall effect of the chemical mixture. However, BKMR often requires a large sample size due to its non-parametric property $[24,70,72]$. WQSR is another supervised mixture analysis method which estimates an empirically weighted index of all pollutants in the mixture. WQSR reduces the impact of outliers by transforming continuous exposures into quantiles. In addition to evaluating joint impacts of multiple pollutants on an outcome, WQSR can be utilized as a variable selection tool, as weights are assigned to each mixture member and reflect the contribution of each mixture component. An important limitation of WQSR is that, unlike BKMR, interactions must be hard-coded into models using prior information. WQSR also assumes directional homogeneity in the mixtures effect (i.e., all mixture components are either positively or negatively associated with the outcome), which may not be a reasonable assumption when both toxic and essential metals are being evaluated. It also assumed linear exposure-response relationships and non-additivity $[25,70,73]$. The strong assumptions of WQSR make it less flexible than BKMR, but it is also less computationally intensive and can be very useful in situations where those assumptions can be met. Of note, quantile g-computation, a newer approach, relaxes the assumptions of directional homogeneity, linearity, and additive relationships [26]. This method was also shown to be more robust and to perform better than WQSR in a range of simulation studies [26,74]. 
Despite the multiple existing mixtures analysis techniques, no single method can answer all questions and there is no gold standard for quantifying health effects of environmental chemical mixtures [69,70]. For instance, among the included studies using both BKMR and WQSR, Liu et al. [68] showed that these mixtures analysis methods identified a similar set of metals as the major contributors for risk of $\mathrm{AD}$, whereas Li et al. [53] and Shih et al. [57] observed that these approaches selected very different sets of metals as predictors of lipid markers or BP, respectively. Therefore, well-defined research questions should guide the selection of the best statistical method for analyzing the effect of chemical mixtures. For complex research questions that cannot be addressed by a single mixture analysis technique, more than one method might be employed, maximizing the different strengths of each tool. As this area of methods development continues to rapidly evolve, it is also critical to monitor the availability of new methods [29]. Potential bias amplification due to mutual adjustment for exposure components within a mixture is also an important consideration for environmental mixture studies [75]. Solutions for minimizing co-exposure amplification bias include examining the correlation structure of the exposures prior to the analysis and employing an instrumental variable approach if needed.

\subsection{Challenges and Opportunities in the Study of Metal Mixtures and CVD Risk Factors and Outcomes}

Several opportunities and challenges for future studies should be noted. First, most studies on this topic have been conducted in either the USA or China. To enhance generalizability and explore potentially different dose-response relationships, research should be extended to lower income countries where metal concentrations and exposure sources often differ [76]. Second, while approximately one third of the retrieved studies were conducted in prospective cohort studies, many included studies were cross-sectional in design. These studies may be subject to reverse causation. For example, hypertension increases risk for chronic kidney disease [77], and renal dysfunction in turn may affect the urinary excretion of metals [78]. Therefore, future prospective studies evaluating metal mixture impacts on CVD risk factors and outcomes are strongly recommended. Third, few studies have investigated the impact of metal mixtures on cardiovascular outcomes beyond BP and dyslipidemia. This may be due, in part, to the logistical ease of studies with short duration of follow-up or due to a lack of understanding of the etiologically relevant exposure window for outcomes such as MI or stroke. Regardless, further research is warranted to assess the association between metal mixtures exposure and risk of CVDs less frequently explored, and findings from the current review provides a strong foundation from which to generate new hypotheses. Fourth, to account for potential intra-individual variation in metal concentrations and identify windows of susceptibility, repeated measures of multiple metal exposures, which has rarely been considered, is important and recommended for future studies [4]. Fifth, several of the studies in our review included non-metal exposures in their mixtures $[38,43,51,54]$. While some of these studies observed significant associations between metal exposures and CVD risk factors and outcomes after accounting for other chemical co-exposures [43,51,54], an exposome study which evaluated 89 different chemicals did not find evidence for an association between metal mixtures and childhood BP [38]. Given these findings, and the potentially complicated interactions between metal and non-metal exposures, future studies may also consider other relevant chemical or non-chemical exposures within their mixtures. New analysis methods which are both reproducible and efficient are also greatly needed to accommodate the growing body of biomedical data, including high-dimensional exposome data [23].

Importantly, many of the studies included in this review may have been impacted by residual confounding. Age, sex, and smoking status are key covariates that need to be considered when evaluating metal mixture impacts on risk for CVDs [17]. While most of the included studies adjusted for age and sex, many studies did not account for smoking status. Fish consumption is also an important potential confounder, as certain metals and metal species such as $\mathrm{Hg}$ and arsenobetaine are abundant in fish and seafood [79], and fish also contain essential nutrients that may reduce the risk of CVDs [80,81]. Sensitivity 
analyses restricting to non-fish consumers are therefore important for studies assessing the cardiotoxicity of metal mixtures, especially when arsenic and mercury are being evaluated [82]. Furthermore, while total As concentrations in urine and blood are frequently used as biomarkers of As exposure [83], this measure may largely reflect organic arsenicals in populations which consume fish and seafood intake, rather than inorganic arsenic [84,85]. Speciation of arsenic and/or the utilization of biospecimens which primarily reflect inorganic arsenic, such as toenail clippings, is therefore critical for studies evaluating arsenic as a mixture component [86]. Careful biospecimen selection is also essential for studies examining $\mathrm{Hg}$ exposure within their mixtures, as $\mathrm{Hg}$ concentrations in blood, hair, and nails predominantly reflect methyl $\mathrm{Hg}$ from fish and seafood consumption [87-90], whereas urinary $\mathrm{Hg}$ primarily reflects inorganic $\mathrm{Hg}[91,92]$.

A few of the included studies conducted stratified analysis by potential effect modifiers, including smoking status [48], race [47], and child sex [57]. Identification of effect modification in epidemiological studies can lead to the discovery of biological mechanisms underlying the associations of interest and the development of more targeted interventions [93]. Some of the included studies also reported potential interactions among the metals within the mixture, such as $\mathrm{Cd}-\mathrm{Zn}[53,59]$ or between toxic and essential metal groups [61]. Knowledge of potential metal interactive effects can be beneficial for developing future interventions. For example, $\mathrm{Zn}$-an essential metal—can alleviate Cd's pathogenic impacts $[94,95]$. Recent advancements in mixture analysis methods have enabled estimating and testing interactions among exposures in their effects on outcomes [23].

Finally, to the best of our knowledge, there is currently no meta-analysis technique which can combine findings from multipollutant studies. However, given the growing literature in this field, the development of meta-analysis methods for studies investigating the health impacts of chemical mixtures should be a future research priority.

\section{Conclusions}

To our knowledge, this is the first scoping review of the literature investigating metal mixture impacts on CVD risk factors and outcomes. CVDs are the leading cause of morbidity and mortality worldwide and are anticipated to remain so in the coming decades [96]. Given the potential cardiotoxicity of metal exposures, understanding the role of metal mixture exposures in the development of CVDs is of public health importance. Recognizing the importance of studying complex mixtures of environmental chemicals and their effects on human health, the NIEHS has developed the Combined Exposure and Mixtures Working Group [97] and the PRIME program [23,31]. This increased focus and dedication of resources has helped the field of environmental epidemiology unravel the more complete mechanisms underlying the cardiotoxicity of metal mixtures [97]. The collective evidence currently suggests possible cardiotoxic effects of multiple metals, with many studies identifying important differences between single-pollutant and multipollutant models. However, given that meta-analysis methods for multipollutant studies do not currently exist, and considering important limitations of the original studies, such as a lack of repeated measures of metal mixtures, our conclusions need to be interpreted with caution. Although studying the cardiotoxicity of metal mixture exposures is challenging, it is critical as most individuals are exposed simultaneously to multiple metals; studies utilizing multipollutant models can therefore help policy makers set up more appropriate, evidence-based CVD prevention strategies and standards to reduce metal exposures. There is therefore a need for additional high-quality prospective studies investigating the impacts of metal mixture exposures on CVD risks in populations exposed to a wider range of metal exposures, as well as studies with repeated measures of multiple metal exposures to identify key windows of susceptibility.

Supplementary Materials: The following supporting information can be downloaded at: https: / /www.mdpi.com/article/10.3390/toxics10030116/s1, Table S1: Search strategy; Table S2: Metal concentrations from the studies included in this review; Table S3: Comparisons of the mixtures analysis methods most commonly used in this review. 
Author Contributions: Conceptualization, G.Y., C.G.H. and M.E.R.; Methodology, G.Y.; Formal Analysis, G.Y.; Investigation, G.Y., Y.W., C.G.H. and M.E.R.; Writing-Original Draft Preparation, G.Y., Y.W., C.G.H. and M.E.R.; Writing-Review \& Editing, G.Y., Y.W., C.G.H. and M.E.R.; Visualization, G.Y.; Supervision, C.G.H. and M.E.R.; Funding Acquisition, C.G.H. and M.E.R. All authors have read and agreed to the published version of the manuscript.

Funding: C.G.H. is supported by a K99/R00 Pathway to Independence Award from NIEHS: R00 ES030400. M.E.R. was supported in part by funding from the NIH National Institute of General Medicine P20 GM104416. The Sponsor had no role in the conduct, design or interpretation of the data in this study. The content is solely the responsibility of the authors and does not necessarily represent the official views of the National Institutes of Health.

Institutional Review Board Statement: Not applicable.

Informed Consent Statement: Not applicable.

Data Availability Statement: Not applicable.

Acknowledgments: We would like to thank Pamela J. Bagley at the Biomedical Libraries, Dartmouth College, Hanover, NH, United States for her assistance in the literature search.

Conflicts of Interest: The authors declare no conflict of interest.

\section{Abbreviations}

List of metal components within the mixtures included in this study.

$\begin{array}{ll}\mathrm{Al} & \text { Aluminum } \\ \mathrm{As} & \text { Arsenic } \\ \mathrm{B} & \text { Boron } \\ \mathrm{Ba} & \text { Barium } \\ \mathrm{Be} & \text { Beryllium } \\ \mathrm{Bi} & \text { Bismuth } \\ \mathrm{Cd} & \text { Cadmium } \\ \mathrm{Co} & \text { Cobalt } \\ \mathrm{Cr} & \text { Chromium } \\ \mathrm{CS} & \text { Cesium } \\ \mathrm{Cu} & \text { Copper } \\ \mathrm{Fe} & \text { Iron } \\ \mathrm{Hg} & \text { Mercury } \\ \mathrm{I} & \text { Iodine } \\ \mathrm{Li} & \text { Lithium } \\ \mathrm{Mg} & \text { Magnesium } \\ \mathrm{Mn} & \text { Manganese } \\ \mathrm{Mo} & \text { Molybdenum } \\ \mathrm{Ni} & \text { Nickel } \\ \mathrm{Pb} & \text { Lead } \\ \mathrm{Rb} & \text { Rubidium } \\ \mathrm{Sb} & \text { Antimony } \\ \mathrm{Se} & \text { Selenium } \\ \mathrm{Sn} & \text { Tin } \\ \mathrm{Sr} & \text { Strontium } \\ \mathrm{Ti} & \text { Titanium } \\ \mathrm{Tl} & \text { Thallium } \\ \mathrm{U} & \text { Uranium } \\ \mathrm{V} & \text { Vanadium } \\ \mathrm{W} & \text { Tungsten } \\ \mathrm{Zn} & \text { Zinc } \\ & \end{array}$

List of key abbreviations used in Table 2. 


$\begin{array}{ll}\begin{array}{ll}\text { Study designs } \\ \text { CC }\end{array} & \text { Case-control study; } \\ \text { CCO } & \text { Case-cohort study; } \\ \text { CO } & \text { Prospective cohort study; } \\ \text { CS } & \text { Cross-sectional study } \\ \text { Mixture analysis methods } & \\ \text { AENET-I } & \text { Adaptive elastic-net with main effects and pairwise interactions } \\ \text { BKMR } & \text { Bayesian kernel machine regression } \\ \text { BKMR-P } & \text { Probit extension of Bayesian kernel machine regression } \\ \text { DSA } & \text { Deletion-substitution-addition algorithm } \\ \text { ERS } & \text { Environmental risk score } \\ \text { EWAS } & \text { Environment-wide association Study } \\ \text { LASSO } & \text { Least absolute shrinkage and selection operator } \\ \text { PCA } & \text { Principal component analysis } \\ \text { WQSR } & \text { Weighted quantile sum regression }\end{array}$

\section{References}

1. Cardiovascular Diseases. Available online: https://www.who.int/westernpacific/health-topics/cardiovascular-diseases (accessed on 9 September 2021).

2. Cardiovascular Disease, Chronic Kidney Disease, and Diabetes Mortality Burden of Cardio-Metabolic Risk Factors between 1980 and 2010: Comparative Risk Assessment. Lancet Diabetes Endocrinol. 2014, 2, 634-647. [CrossRef]

3. Briffa, J.; Sinagra, E.; Blundell, R. Heavy Metal Pollution in the Environment and Their Toxicological Effects on Humans. Heliyon 2020, 6, e04691. [CrossRef] [PubMed]

4. $\quad$ Chowdhury, R.; Ramond, A.; O'Keeffe, L.M.; Shahzad, S.; Kunutsor, S.K.; Muka, T.; Gregson, J.; Willeit, P.; Warnakula, S.; Khan, H.; et al. Environmental Toxic Metal Contaminants and Risk of Cardiovascular Disease: Systematic Review and MetaAnalysis. BMJ 2018, 362, k3310. [CrossRef] [PubMed]

5. Alissa, E.M.; Ferns, G.A. Heavy Metal Poisoning and Cardiovascular Disease. J. Toxicol. 2011, 2011, 870125. [CrossRef] [PubMed]

6. Yang, D.; Yang, Q.; Fu, N.; Li, S.; Han, B.; Liu, Y.; Tang, Y.; Guo, X.; Lv, Z.; Zhang, Z. Hexavalent Chromium Induced Heart Dysfunction via Sesn2-Mediated Impairment of Mitochondrial Function and Energy Supply. Chemosphere 2021, $264,128547$. [CrossRef] [PubMed]

7. Hudson, K.M.; Belcher, S.M.; Cowley, M. Maternal Cadmium Exposure in the Mouse Leads to Increased Heart Weight at Birth and Programs Susceptibility to Hypertension in Adulthood. Sci. Rep. 2019, 9, 13553. [CrossRef]

8. Solenkova, N.V.; Newman, J.D.; Berger, J.S.; Thurston, G.; Hochman, J.S.; Lamas, G.A. Metal Pollutants and Cardiovascular Disease: Mechanisms and Consequences of Exposure. Am. Heart J. 2014, 168, 812-822. [CrossRef]

9. Iavicoli, I.; Fontana, L.; Bergamaschi, A. The Effects of Metals as Endocrine Disruptors. J. Toxicol. Environ. Health B Crit. Rev. 2009, 12, 206-223. [CrossRef]

10. Huang, Y.-C.T.; Ghio, A.J. Vascular Effects of Ambient Pollutant Particles and Metals. Curr. Vasc. Pharmacol. 2006, 4, 199-203. [CrossRef]

11. Omanwar, S.; Fahim, M. Mercury Exposure and Endothelial Dysfunction: An Interplay Between Nitric Oxide and Oxidative Stress. Int. J. Toxicol. 2015, 34, 300-307. [CrossRef]

12. Prozialeck, W.C.; Edwards, J.R.; Nebert, D.W.; Woods, J.M.; Barchowsky, A.; Atchison, W.D. The Vascular System as a Target of Metal Toxicity. Toxicol. Sci. 2008, 102, 207-218. [CrossRef] [PubMed]

13. Ruiz-Hernandez, A.; Kuo, C.-C.; Rentero-Garrido, P.; Tang, W.-Y.; Redon, J.; Ordovas, J.M.; Navas-Acien, A.; Tellez-Plaza, M. Environmental Chemicals and DNA Methylation in Adults: A Systematic Review of the Epidemiologic Evidence. Clin. Epigenet. 2015, 7, 55. [CrossRef] [PubMed]

14. Lee, D.-H.; Jacobs, D.R.; Porta, M. Hypothesis: A Unifying Mechanism for Nutrition and Chemicals as Lifelong Modulators of DNA Hypomethylation. Environ. Health Perspect. 2009, 117, 1799-1802. [CrossRef]

15. Chia, N.; Wang, L.; Lu, X.; Senut, M.-C.; Brenner, C.; Ruden, D.M. Hypothesis: Environmental Regulation of 5-Hydroxymethylcytosine by Oxidative Stress. Epigenetics 2011, 6, 853-856. [CrossRef] [PubMed]

16. Dai, H.; Wang, Z. Histone Modification Patterns and Their Responses to Environment. Curr. Environ. Health Rep. 2014, 1, 11-21. [CrossRef]

17. Nigra, A.E.; Ruiz-Hernandez, A.; Redon, J.; Navas-Acien, A.; Tellez-Plaza, M. Environmental Metals and Cardiovascular Disease in Adults: A Systematic Review beyond Lead and Cadmium. Curr. Environ. Health Rep. 2016, 3, 416-433. [CrossRef]

18. Hu, X.F.; Lowe, M.; Chan, H.M. Mercury Exposure, Cardiovascular Disease, and Mortality: A Systematic Review and DoseResponse Meta-Analysis. Environ. Res. 2021, 193, 110538. [CrossRef]

19. Gibson, E.A.; Nunez, Y.; Abuawad, A.; Zota, A.R.; Renzetti, S.; Devick, K.L.; Gennings, C.; Goldsmith, J.; Coull, B.A.; Kioumourtzoglou, M.-A. An Overview of Methods to Address Distinct Research Questions on Environmental Mixtures: An Application to Persistent Organic Pollutants and Leukocyte Telomere Length. Environ. Health 2019, 18, 76. [CrossRef] 
20. Chemical Mixtures in Environmental Health (1998) | RFA Text | Research Project Database | Grantee Research Project | ORD | US EPA. Available online: https://cfpub.epa.gov/ncer_abstracts/index.cfm/fuseaction/display.rfatext/rfa_id/99 (accessed on 21 February 2022).

21. Carlin, D.J.; Rider, C.V.; Woychik, R.; Birnbaum, L.S. Unraveling the Health Effects of Environmental Mixtures: An NIEHS Priority. Environ. Health Perspect. 2013, 121, A6-A8. [CrossRef]

22. Rider, C.V.; Carlin, D.J.; Devito, M.J.; Thompson, C.L.; Walker, N.J. Mixtures Research at NIEHS: An Evolving Program. Toxicology 2013, 313, 94-102. [CrossRef]

23. Joubert, B.R.; Kioumourtzoglou, M.-A.; Chamberlain, T.; Chen, H.Y.; Gennings, C.; Turyk, M.E.; Miranda, M.L.; Webster, T.F.; Ensor, K.B.; Dunson, D.B.; et al. Powering Research through Innovative Methods for Mixtures in Epidemiology (PRIME) Program: Novel and Expanded Statistical Methods. Int. J. Environ. Res. Public Health 2022, 19, 1378. [CrossRef] [PubMed]

24. Bobb, J.F.; Claus Henn, B.; Valeri, L.; Coull, B.A. Statistical Software for Analyzing the Health Effects of Multiple Concurrent Exposures via Bayesian Kernel Machine Regression. Environ. Health 2018, 17, 67. [CrossRef] [PubMed]

25. Carrico, C.; Gennings, C.; Wheeler, D.C.; Factor-Litvak, P. Characterization of Weighted Quantile Sum Regression for Highly Correlated Data in a Risk Analysis Setting. JABES 2015, 20, 100-120. [CrossRef] [PubMed]

26. Keil, A.P.; Buckley, J.P.; O’Brien, K.M.; Ferguson, K.K.; Zhao, S.; White, A.J. A Quantile-Based g-Computation Approach to Addressing the Effects of Exposure Mixtures. Environ. Health Perspect. 2020, 128, 047004. [CrossRef] [PubMed]

27. Tricco, A.C.; Lillie, E.; Zarin, W.; O’Brien, K.K.; Colquhoun, H.; Levac, D.; Moher, D.; Peters, M.D.J.; Horsley, T.; Weeks, L.; et al. PRISMA Extension for Scoping Reviews (PRISMA-ScR): Checklist and Explanation. Ann. Intern. Med. 2018, 169, 467-473. [CrossRef]

28. Yim, G. Exposure to Metal Mixtures in Association with Cardiovascular Risk Factors and Outcomes: A Scoping Review. 2021. Available online: https: / osf.io/hbufn/ (accessed on 16 January 2022). [CrossRef]

29. Taylor, K.W.; Joubert, B.R.; Braun, J.M.; Dilworth, C.; Gennings, C.; Hauser, R.; Heindel, J.J.; Rider, C.V.; Webster, T.F.; Carlin, D.J. Statistical Approaches for Assessing Health Effects of Environmental Chemical Mixtures in Epidemiology: Lessons from an Innovative Workshop. Environ. Health Perspect. 2016, 124, A227-A229. [CrossRef]

30. Morgan, R.L.; Whaley, P.; Thayer, K.A.; Schünemann, H.J. Identifying the PECO: A Framework for Formulating Good Questions to Explore the Association of Environmental and Other Exposures with Health Outcomes. Environ. Int. 2018, 121, 1027-1031. [CrossRef]

31. RFA-ES-17-001: Powering Research through Innovative Methods for Mixtures in Epidemiology (PRIME) (R01). Available online: https:/ / grants.nih.gov/grants/guide/rfa-files/RFA-ES-17-001.html (accessed on 18 February 2022).

32. Schuster, N.A.; Hoogendijk, E.O.; Kok, A.A.L.; Twisk, J.W.R.; Heymans, M.W. Ignoring Competing Events in the Analysis of Survival Data May Lead to Biased Results: A Nonmathematical Illustration of Competing Risk Analysis. J. Clin. Epidemiol. 2020, 122, 42-48. [CrossRef]

33. Wolffe, T.A.M.; Whaley, P.; Halsall, C.; Rooney, A.A.; Walker, V.R. Systematic Evidence Maps as a Novel Tool to Support Evidence-Based Decision-Making in Chemicals Policy and Risk Management. Environ. Int. 2019, 130, 104871. [CrossRef]

34. Munn, Z.; Peters, M.D.J.; Stern, C.; Tufanaru, C.; McArthur, A.; Aromataris, E. Systematic Review or Scoping Review? Guidance for Authors When Choosing between a Systematic or Scoping Review Approach. BMC Med. Res. Methodol. 2018, 18, 143. [CrossRef]

35. Dai, L.; Koutrakis, P.; Coull, B.A.; Sparrow, D.; Vokonas, P.S.; Schwartz, J.D. Use of the Adaptive LASSO Method to Identify PM2.5 Components Associated with Blood Pressure in Elderly Men: The Veterans Affairs Normative Aging Study. Environ. Health Perspect. 2016, 124, 120-125. [CrossRef] [PubMed]

36. Xu, J.; White, A.J.; Niehoff, N.M.; O’Brien, K.M.; Sandler, D.P. Airborne Metals Exposure and Risk of Hypertension in the Sister Study. Environ. Res. 2020, 191, 110144. [CrossRef] [PubMed]

37. Karakis, I.; Baumfeld, Y.; Landau, D.; Gat, R.; Shemesh, N.; Yitshak-Sade, M.; Tirosh, O.; Sarov, B.; Novack, L. Exposure to Metals and Morbidity at Eight Years Follow-up in Women of Childbearing Age. Sci. Rep. 2021, 11, 11429. [CrossRef] [PubMed]

38. Warembourg, C.; Maitre, L.; Tamayo-Uria, I.; Fossati, S.; Roumeliotaki, T.; Aasvang, G.M.; Andrusaityte, S.; Casas, M.; Cequier, E.; Chatzi, L.; et al. Early-Life Environmental Exposures and Blood Pressure in Children. J. Am. Coll. Cardiol. 2019, 74, 1317-1328. [CrossRef]

39. Castiello, F.; Olmedo, P.; Gil, F.; Molina, M.; Mundo, A.; Romero, R.R.; Ruíz, C.; Gómez-Vida, J.; Vela-Soria, F.; Freire, C. Association of Urinary Metal Concentrations with Blood Pressure and Serum Hormones in Spanish Male Adolescents. Environ. Res. 2020, 182, 108958. [CrossRef]

40. Bulka, C.M.; Bryan, M.S.; Persky, V.W.; Daviglus, M.L.; Durazo-Arvizu, R.A.; Parvez, F.; Slavkovich, V.; Graziano, J.H.; Islam, T.; Baron, J.A.; et al. Changes in Blood Pressure Associated with Lead, Manganese, and Selenium in a Bangladeshi Cohort. Environ. Pollut. 2019, 248, 28-35. [CrossRef]

41. Elongi Moyene, J.-P.; Scheers, H.; Tandu-Umba, B.; Haufroid, V.; Buassa-bu-Tsumbu, B.; Verdonck, F.; Spitz, B.; Nemery, B. Preeclampsia and Toxic Metals: A Case-Control Study in Kinshasa, DR Congo. Environ. Health 2016, 15, 48. [CrossRef]

42. Park, S.K.; Zhao, Z.; Mukherjee, B. Construction of Environmental Risk Score beyond Standard Linear Models Using Machine Learning Methods: Application to Metal Mixtures, Oxidative Stress and Cardiovascular Disease in NHANES. Environ. Health 2017, 16, 102. [CrossRef] 
43. Park, S.K.; Tao, Y.; Meeker, J.D.; Harlow, S.D.; Mukherjee, B. Environmental Risk Score as a New Tool to Examine Multi-Pollutants in Epidemiologic Research: An Example from the NHANES Study Using Serum Lipid Levels. PLoS ONE 2014, 9 , e98632. [CrossRef]

44. Yao, X.; Steven Xu, X.; Yang, Y.; Zhu, Z.; Zhu, Z.; Tao, F.; Yuan, M. Stratification of Population in NHANES 2009-2014 Based on Exposure Pattern of Lead, Cadmium, Mercury, and Arsenic and Their Association with Cardiovascular, Renal and Respiratory Outcomes. Environ. Int. 2021, 149, 106410. [CrossRef]

45. Wang, X.; Mukherjee, B.; Park, S.K. Associations of Cumulative Exposure to Heavy Metal Mixtures with Obesity and Its Comorbidities Among U.S. Adults in NHANES 2003-2014. Environ. Int. 2018, 121, 683-694. [CrossRef] [PubMed]

46. Desai, G.; Niu, Z.; Luo, W.; Frndak, S.; Shaver, A.L.; Kordas, K. Low-Level Exposure to Lead, Mercury, Arsenic, and Cadmium, and Blood Pressure among 8-17-Year-Old Participants of the 2009-2016 National Health and Nutrition Examination Survey. Environ. Res. 2021, 197, 111086. [CrossRef] [PubMed]

47. Everson, T.M.; Niedzwiecki, M.M.; Toth, D.; Tellez-Plaza, M.; Liu, H.; Barr, D.B.; Gribble, M.O. Metal Biomarker Mixtures and Blood Pressure in the United States: Cross-Sectional Findings from the 1999-2006 National Health and Nutrition Examination Survey (NHANES). Environ. Health 2021, 20, 15. [CrossRef]

48. Yang, L.; Jiang, Y.; Zhao, L.; Li, M.; Guo, W.; Shu, J.; Zhu, R.; Zhang, X. Multiple Metals Exposure and Arterial Stiffness: A Panel Study in China. Chemosphere 2021, 263, 128217. [CrossRef]

49. Kim, K.; Park, H. Co-Exposure to Heavy Metals and Hypertension Among Adults in South Korea. Expo. Health 2021. [CrossRef]

50. Xu, J.; Engel, L.S.; Rhoden, J.; Jackson, W.B.; Kwok, R.K.; Sandler, D.P. The Association between Blood Metals and Hypertension in the GuLF Study. Environ. Res. 2021, 202, 111734. [CrossRef] [PubMed]

51. Zuk, A.M.; Liberda, E.N.; Tsuji, L.J.S. Environmental Contaminant Body Burdens and the Relationship with Blood Pressure Measures Among Indigenous Adults. Environ. Epidemiol. 2021, 5, e137. [CrossRef]

52. Zhu, X.; Fan, Y.; Sheng, J.; Gu, L.; Tao, Q.; Huang, R.; Liu, K.; Yang, L.; Chen, G.; Cao, H.; et al. Association Between Blood Heavy Metal Concentrations and Dyslipidemia in the Elderly. Biol. Trace Elem. Res. 2021, 199, 1280-1290. [CrossRef]

53. Li, Z.; Xu, Y.; Huang, Z.; Wei, Y.; Hou, J.; Long, T.; Wang, F.; Cheng, X.; Duan, Y.; Chen, X.; et al. Association of Multiple Metals with Lipid Markers against Different Exposure Profiles: A Population-Based Cross-Sectional Study in China. Chemosphere 2021, 264, 128505. [CrossRef]

54. Liberda, E.N.; Zuk, A.M.; Tsuji, L.J.S. Complex Contaminant Mixtures and Their Associations with Intima-Media Thickness. BMC Cardiovasc. Disord 2019, 19, 289. [CrossRef]

55. Kupsco, A.; Kioumourtzoglou, M.-A.; Just, A.C.; Amarasiriwardena, C.; Estrada-Gutierrez, G.; Cantoral, A.; Sanders, A.P.; Braun, J.M.; Svensson, K.; Brennan, K.J.M.; et al. Prenatal Metal Concentrations and Childhood Cardiometabolic Risk Using Bayesian Kernel Machine Regression to Assess Mixture and Interaction Effects. Epidemiology 2019, 30, 263-273. [CrossRef] [PubMed]

56. Howe, C.G.; Margetaki, K.; Vafeiadi, M.; Roumeliotaki, T.; Karachaliou, M.; Kogevinas, M.; McConnell, R.; Eckel, S.P.; Conti, D.V.; Kippler, M.; et al. Prenatal Metal Mixtures and Child Blood Pressure in the Rhea Mother-Child Cohort in Greece. Environ. Health 2021, 20, 1. [CrossRef] [PubMed]

57. Shih, Y.-H.; Howe, C.G.; Scannell Bryan, M.; Shahriar, M.; Kibriya, M.G.; Jasmine, F.; Sarwar, G.; Graziano, J.H.; Persky, V.W.; Jackson, B.; et al. Exposure to Metal Mixtures in Relation to Blood Pressure among Children 5-7 Years Old. Environ. Epidemiol. 2021, 5, e135. [CrossRef] [PubMed]

58. Zhang, M.; Liu, T.; Wang, G.; Buckley, J.P.; Guallar, E.; Hong, X.; Wang, M.-C.; Wills-Karp, M.; Wang, X.; Mueller, N.T. In Utero Exposure to Heavy Metals and Trace Elements and Childhood Blood Pressure in a U.S. Urban, Low-Income, Minority Birth Cohort. Environ. Health Perspect. 2021, 129, 067005. [CrossRef]

59. Zhong, Q.; Wu, H.; Niu, Q.; Jia, P.; Qin, Q.; Wang, X.; He, J.; Yang, W.; Huang, F. Exposure to Multiple Metals and the Risk of Hypertension in Adults: A Prospective Cohort Study in a Local Area on the Yangtze River, China. Environ. Int. 2021, 153, 106538. [CrossRef]

60. Jiang, Q.; Xiao, Y.; Long, P.; Li, W.; Yu, Y.; Liu, Y.; Liu, K.; Zhou, L.; Wang, H.; Yang, H.; et al. Associations of Plasma Metal Concentrations with Incident Dyslipidemia: Prospective Findings from the Dongfeng-Tongji Cohort. Chemosphere 2021, 285, 131497. [CrossRef]

61. Bommarito, P.A.; Kim, S.S.; Meeker, J.D.; Fry, R.C.; Cantonwine, D.E.; McElrath, T.F.; Ferguson, K.K. Urinary Trace Metals, Maternal Circulating Angiogenic Biomarkers, and Preeclampsia: A Single-Contaminant and Mixture-Based Approach. Environ. Health 2019, 18, 63. [CrossRef]

62. Liu, T.; Zhang, M.; Rahman, M.L.; Wang, X.; Hinkle, S.N.; Zhang, C.; Mueller, N.T. Exposure to Heavy Metals and Trace Minerals in First Trimester and Maternal Blood Pressure Change over Gestation. Environ. Int. 2021, 153, 106508. [CrossRef]

63. Domingo-Relloso, A.; Grau-Perez, M.; Briongos-Figuero, L.; Gomez-Ariza, J.L.; Garcia-Barrera, T.; Dueñas-Laita, A.; Bobb, J.F.; Chaves, F.J.; Kioumourtzoglou, M.-A.; Navas-Acien, A.; et al. The Association of Urine Metals and Metal Mixtures with Cardiovascular Incidence in an Adult Population from Spain: The Hortega Follow-Up Study. Int. J. Epidemiol. 2019, 48, 1839-1849. [CrossRef]

64. Wang, Y.; Wang, K.; Han, T.; Zhang, P.; Chen, X.; Wu, W.; Feng, Y.; Yang, H.; Li, M.; Xie, B.; et al. Exposure to Multiple Metals and Prevalence for Preeclampsia in Taiyuan, China. Environ. Int. 2020, 145, 106098. [CrossRef] 
65. Wen, Y.; Huang, S.; Zhang, Y.; Zhang, H.; Zhou, L.; Li, D.; Xie, C.; Lv, Z.; Guo, Y.; Ke, Y.; et al. Associations of Multiple Plasma Metals with the Risk of Ischemic Stroke: A Case-Control Study. Environ. Int. 2019, 125, 125-134. [CrossRef]

66. Xiao, Y.; Yuan, Y.; Liu, Y.; Yu, Y.; Jia, N.; Zhou, L.; Wang, H.; Huang, S.; Zhang, Y.; Yang, H.; et al. Circulating Multiple Metals and Incident Stroke in Chinese Adults. Stroke 2019, 50, 1661-1668. [CrossRef] [PubMed]

67. Cabral, M.; Kuxhaus, O.; Eichelmann, F.; Kopp, J.F.; Alker, W.; Hackler, J.; Kipp, A.P.; Schwerdtle, T.; Haase, H.; Schomburg, L.; et al. Trace Element Profile and Incidence of Type 2 Diabetes, Cardiovascular Disease and Colorectal Cancer: Results from the EPIC-Potsdam Cohort Study. Eur. J. Nutr. 2021, 60, 3267-3278. [CrossRef]

68. Liu, Q.; Jin, J.; Xu, C.; Li, W.; Liang, J.; Xu, J.; Weng, Z.; Zhang, X.; Zhang, X.; Shao, J.; et al. HDL Cholesterol: A Potential Mediator of the Association between Serum Levels of a Mixture of Metals and the Risk of Aortic Dissection in a Chinese Population. Environ. Pollut. 2021, 290, 117942. [CrossRef] [PubMed]

69. Vuong, A.M.; Yolton, K.; Braun, J.M.; Lanphear, B.P.; Chen, A. Chemical Mixtures and Neurobehavior: A Review of Epidemiologic Findings and Future Directions. Rev. Environ. Health 2020, 35, 245-256. [CrossRef] [PubMed]

70. Gibson, E.A.; Goldsmith, J.; Kioumourtzoglou, M.-A. Complex Mixtures, Complex Analyses: An Emphasis on Interpretable Results. Curr. Environ. Health Rep. 2019, 6, 53-61. [CrossRef] [PubMed]

71. Stafoggia, M.; Breitner, S.; Hampel, R.; Basagaña, X. Statistical Approaches to Address Multi-Pollutant Mixtures and Multiple Exposures: The State of the Science. Curr. Environ. Health Rep. 2017, 4, 481-490. [CrossRef] [PubMed]

72. Bobb, J.F.; Valeri, L.; Claus Henn, B.; Christiani, D.C.; Wright, R.O.; Mazumdar, M.; Godleski, J.J.; Coull, B.A. Bayesian Kernel Machine Regression for Estimating the Health Effects of Multi-Pollutant Mixtures. Biostatistics 2015, 16, 493-508. [CrossRef]

73. Gennings, C.; Carrico, C.; Factor-Litvak, P.; Krigbaum, N.; Cirillo, P.M.; Cohn, B.A. A Cohort Study Evaluation of Maternal PCB Exposure Related to Time to Pregnancy in Daughters. Environ. Health 2013, 12, 66. [CrossRef]

74. Schmidt, S. Quantile G-Computation: A New Method for Analyzing Mixtures of Environmental Exposures. Environ. Health Perspect. 2020, 128, 104004. [CrossRef]

75. Weisskopf, M.G.; Seals, R.M.; Webster, T.F. Bias Amplification in Epidemiologic Analysis of Exposure to Mixtures. Environ. Health Perspect. 2018, 126, 047003. [CrossRef] [PubMed]

76. Anyanwu, B.O.; Ezejiofor, A.N.; Igweze, Z.N.; Orisakwe, O.E. Heavy Metal Mixture Exposure and Effects in Developing Nations: An Update. Toxics 2018, 6, 65. [CrossRef]

77. Said, S.; Hernandez, G.T. The Link between Chronic Kidney Disease and Cardiovascular Disease. J. Nephropathol. 2014, 3, 99-104. [CrossRef]

78. Yang, A.-M.; Lo, K.; Zheng, T.-Z.; Yang, J.-L.; Bai, Y.-N.; Feng, Y.-Q.; Cheng, N.; Liu, S.-M. Environmental Heavy Metals and Cardiovascular Diseases: Status and Future Direction. Chronic Dis. Transl. Med. 2020, 6, 251-259. [CrossRef] [PubMed]

79. Bosch, A.C.; O’Neill, B.; Sigge, G.O.; Kerwath, S.E.; Hoffman, L.C. Heavy Metals in Marine Fish Meat and Consumer Health: A Review. J. Sci. Food Agric. 2016, 96, 32-48. [CrossRef] [PubMed]

80. Mohan, D.; Mente, A.; Dehghan, M.; Rangarajan, S.; O’Donnell, M.; Hu, W.; Dagenais, G.; Wielgosz, A.; Lear, S.; Wei, L.; et al. Associations of Fish Consumption with Risk of Cardiovascular Disease and Mortality Among Individuals With or Without Vascular Disease From 58 Countries. JAMA Intern. Med. 2021, 181, 631-649. [CrossRef] [PubMed]

81. Krittanawong, C.; Isath, A.; Hahn, J.; Wang, Z.; Narasimhan, B.; Kaplin, S.L.; Jneid, H.; Virani, S.S.; Tang, W.H.W. Fish Consumption and Cardiovascular Health: A Systematic Review. Am. J. Med. 2021, 134, 713-720. [CrossRef]

82. Hu, X.F.; Singh, K.; Chan, H.M. Mercury Exposure, Blood Pressure, and Hypertension: A Systematic Review and Dose-Response Meta-Analysis. Environ. Health Perspect. 2018, 126, 076002. [CrossRef]

83. Marchiset-Ferlay, N.; Savanovitch, C.; Sauvant-Rochat, M.-P. What Is the Best Biomarker to Assess Arsenic Exposure via Drinking Water? Environ. Int. 2012, 39, 150-171. [CrossRef]

84. Vahter, M. Mechanisms of Arsenic Biotransformation. Toxicology 2002, 181-182, 211-217. [CrossRef]

85. Chou, C.-H.S.J.; Harper, C. Toxicological Profile for Arsenic; United States Agency for Toxic Substances and Disease Registry: Atlanta, GA, USA, 2007.

86. Slotnick, M.J.; Meliker, J.R.; AvRuskin, G.A.; Ghosh, D.; Nriagu, J.O. Toenails as a Biomarker of Inorganic Arsenic Intake from Drinking Water and Foods. J. Toxicol. Environ. Health A 2007, 70, 148-158. [CrossRef] [PubMed]

87. Mercury | Toxicological Profile I ATSDR. Available online: https://wwwn.cdc.gov/TSP/ToxProfiles/ToxProfiles.aspx?id=115\& tid=24 (accessed on 22 February 2022).

88. Clarkson, T.W.; Vyas, J.B.; Ballatori, N. Mechanisms of Mercury Disposition in the Body. Am. J. Ind. Med. 2007, 50, 757-764. [CrossRef] [PubMed]

89. Sakamoto, M.; Chan, H.M.; Domingo, J.L.; Oliveira, R.B.; Kawakami, S.; Murata, K. Significance of Fingernail and Toenail Mercury Concentrations as Biomarkers for Prenatal Methylmercury Exposure in Relation to Segmental Hair Mercury Concentrations. Environ. Res. 2015, 136, 289-294. [CrossRef] [PubMed]

90. Berglund, M.; Lind, B.; Björnberg, K.A.; Palm, B.; Einarsson, O.; Vahter, M. Inter-Individual Variations of Human Mercury Exposure Biomarkers: A Cross-Sectional Assessment. Environ. Health 2005, 4, 20. [CrossRef]

91. Risher, J.F.; De Rosa, C.T.; Jones, D.E.; Murray, H.E. Summary Report for the Expert Panel Review of the Toxicological Profile for Mercury. 1999. Available online: https://journals.sagepub.com/doi/10.1177/074823379901500504 (accessed on 20 February 2022). 
92. Zalups, R.K.; Barfuss, D.W.; Kostyniak, P.J. Altered Intrarenal Accumulation of Mercury in Uninephrectomized Rats Treated with Methylmercury Chloride. Toxicol. Appl. Pharmacol. 1992, 115, 174-182. [CrossRef]

93. Bours, M.J.L. Tutorial: A Nontechnical Explanation of the Counterfactual Definition of Effect Modification and Interaction. J. Clin. Epidemiol. 2021, 134, 113-124. [CrossRef]

94. Yu, H.; Zhen, J.; Leng, J.; Cai, L.; Ji, H.; Keller, B.B. Zinc as a Countermeasure for Cadmium Toxicity. Acta Pharmacol. Sin. 2021, 42, 340-346. [CrossRef]

95. McCarty, M.F. Zinc and Multi-Mineral Supplementation Should Mitigate the Pathogenic Impact of Cadmium Exposure. Med. Hypotheses 2012, 79, 642-648. [CrossRef]

96. Mathers, C.D.; Loncar, D. Projections of Global Mortality and Burden of Disease from 2002 to 2030. PLoS Med. 2006, 3, e442. [CrossRef]

97. Chemical Mixtures. Available online: https://www.niehs.nih.gov/health/topics/science/chemical-mixtures/index.cfm (accessed on 20 February 2022). 